\title{
A COLLOCATION METHOD SOLVING INTEGRAL EQUATION MODELS FOR IMAGE RESTORATION
}

\author{
YUZHEN LIU, LIXIN SHEN, YUESHENG XU AND HONGQI YANG \\ Communicated by $\mathrm{Da} \mathrm{Xu}$
}

\begin{abstract}
We propose a collocation method for solving integral equations which model image restoration from out-of-focus images. Restoration of images from out-of-focus images can be formulated as an integral equation of the first kind, which is an ill-posed problem. We employ the Tikhonov regularization to treat the ill-posedness and obtain results of a well-posed second kind integral equation whose integral operator is the square of the original operator. The present of the square of the integral operator requires high computational cost to solve the equation. To overcome this difficulty, we convert the resulting second kind integral equation into an equivalent system of integral equations which do not involve the square of the integral operator. A multiscale collocation method is then applied to solve the system. A truncation strategy for the matrices appearing in the resulting discrete linear system is proposed to design a fast numerical solver for the system of integral equations. A quadrature method is used to compute the entries of the resulting matrices. We estimate the computational cost of the numerical method and its approximate accuracy. Numerical experiments are presented to demonstrate the performance of the proposed method for image restoration.
\end{abstract}

1. Introduction. Discrete models are usually used in image processing due to their convenience in implementation and their consistence with the usual sampling method. However, since discrete models result from piecewise constant approximation of the integral equation

2010 AMS Mathematics subject classification. Primary 65R20, 65R32.

Keywords and phrases. Image restoration, inverse problems.

This research was partially supported by the Guangdong Provincial Government of China through the Computational Science Innovative Research Team program, by the Natural Science Foundation of China under grant Nos. 11471013, 11571386, 91530117, and by the U.S. National Science Foundation under grant No. DMS1522332. The third author is a Professor Emeritus of Syracuse University. All correspondence should be sent to the third author.

Received by the editors on August 1, 2015, and in revised form on November 10, 2015 .

DOI: $10.1216 /$ JIE-2016-28-2-263

Copyright (C)2016 Rocky Mountain Mathematics Consortium 
which describes the processing, this imposes a bottleneck model error which cannot be compensated for by any image processing method.

To overcome the shortcoming of discrete models, we shall use the continuous model directly instead of an existing discrete model in image restoration. The idea of using a continuous model for image restoration was first proposed in [16]. There are several advantages of using the continuous integral equation as commented upon in [16]. First of all, continuous models are derived directly from physical laws, and therefore, they are physically meaningful and more accurate to represent the physical phenomena. Second, continuous models allow us to discretize them by a numerical method with a higher order of accuracy instead of piecewise constant approximation, and hence the resulting model error can be significantly reduced in comparison to the model error of the piecewise constant discretization, especially in the image enlargement, since an approximate solution obtained from the continuous model is defined at every point in the continuous image domain, interpolation which is required for that obtained from the discrete model is no longer needed. Hence, the continuous model is particularly suitable for image enlargement.

Direct use of a continuous model in image restoration has certain new challenges. In image processing, the related integral operators are compact in appropriate Banach spaces since their kernels are normally smooth. As a result, the solutions of the related first kind integral equations do not continuously depend on given data. Hence, a small perturbation in the given data may cause a large perturbation in the solution of the integral equation. This requires us to use an appropriate regularization method to convert the ill-posed integral equation to a well-posed one. Such ill-posed integral equations of the first kind are usually turned to a class of well-posed Fredholm integral equations of the second kind by a regularization method. We shall use the Tikhonov method [13] to regularize the first kind integral equation. However, the resulting well-posed second kind integral equation involves the square of the original self-adjoint integral operator, and it will cost much more to compute the square of a Fredholm integral operator than to compute the original operator. Following [3], we shall convert the second kind integral equation which involves the square of the integral operator into an equivalent system of integral equations which do not involve 
the square of the integral operator. In this way, we avoid computing the square of the integral operator.

There are two other two crucial issues that remain to be resolved. Appropriate choice of the regularization parameter is crucial for the success of the regularization method. An optimal regularization parameter should give the best balance between the well-posedness and approximation accuracy. The choice strategies of such parameters have been discussed by many researchers (for example, see $[\mathbf{1 3}, \mathbf{1 7}, \mathbf{2 1}, \mathbf{2 4}, \mathbf{2 5}]$ ). Another issue is that discretization of the resulting integral equations of the second kind leads to algebraic systems with full coefficient matrices, and efficient numerical methods are required to reduce the large computational cost in the numerical solutions of the systems. The representation of certain integral operators under a multiscale basis is numerically sparse and thus matrix compression techniques can be used to approximate the full matrix by a sparse matrix. The idea has been used for solving well-posed integral equations, and the methodology of this method was described in [7].

We develop a multiscale piecewise polynomial collocation method for solving this equation and then use the multiscale analysis to develop a matrix compression technique which leads to a fast solver for the integral equations. The reason that we choose the collocation method is its lower computational cost in generating the coefficient matrix of the corresponding discrete equation in comparison with the Galerkin method. As shown in [16], the Galerkin method approximates both the range and domain of the integral operator by the same basis functions and, thus, the entries of the resulting discrete matrix are integrals whose integration dimensions double those of the original integral that defines the operator. However, there is a challenging issue related to developing the collocation method for solving the ill-posed integral equation since a posteriori parameter choice strategies for the fast collocation method demands certain estimates in the $L^{\infty}(E)$ norm, which are not available. This is one of our focuses of this paper. We shall give an estimate of the $L^{\infty}$ norm bound of the regularized integral operator and develop a posteriori parameter choice strategies for the collocation method.

We organize this paper in seven sections. In Section 2, we introduce the integral equation of the first kind that governs the image restoration from an out-of-focus image, consider its Tikhonov regularized equation and convert the Tikhonov regularization equation into a 
system of integral equations which do not involve the square of the original integral operator. We describe in Section 3 a multiscale collocation method based on the multiwavelet of Micchelli and Xu $[\mathbf{1 8}, \mathbf{1 9}]$ and the multiscale collocation functionals of Chen, Micchelli and Xu $[4,6]$ for solving the corresponding regularized integral equation of the second kind. We propose a matrix compression strategy in Section 4. In Section 5, we introduce the numerical quadrature scheme to compute the nonzero entries of the compressed coefficient matrix, which is designed so that the quadrature error will not ruin the overall convergence order and the number of functional evaluations used in computing all the nonzero entries is linear with a logarithmic factor with respect to the dimension of the approximate solution space. We propose in Section 6 a posteriori regularization parameter strategies and provide a complete analysis of the convergence rate of the corresponding approximate solution obtained by using the parameter. Finally, in Section 7, we present numerical examples to demonstrate the performance of the proposed method for image restoration.

2. An integral equation model for image restoration. In this section, we describe an integral equation model for image restoration. In particular, we are interested in the integral equation which governs the image restoration from an out-of-focus image.

The image restoration problem may be modeled by an integral equation of the first kind. Specifically, we let $\Omega \subset R^{2}$ denote the image domain, usually a rectangular region. We are given an observed image $f: \Omega \rightarrow R$ and wish to restore the original image $v: \Omega \rightarrow R$ from $f$. The original image and the observed image are related via the integral equation

$$
\mathcal{G} v=f,
$$

where $\mathcal{G}$ is the Fredholm integral operator defined in terms of a kernel $k$ by

$$
(\mathcal{G} v)(x):=\int_{\Omega} k\left(x, x^{\prime}\right) v\left(x^{\prime}\right) d x^{\prime}, \quad x \in \Omega .
$$

We call $\mathcal{G}$ the blurring operator. Mathematically, restoring the original image from a blurred image $f$ is equivalent to solving $v$ from the integral equation (2.1). 
The choice of the kernel depends on the specific application context. Examples of integral equation (2.1) with special kernel may be found in $[2,14]$. In this paper, we will focus on developing basic concepts of using integral equations in the numerical process of image restoration and, for this reason, we will use the out-of-focus image model as an example. Let $\Omega:=E \times E$ with $E:=[0,1]$. An out-of-focus image is usually modeled [11] with the kernel

$$
k\left(x, x^{\prime}\right)=\frac{1}{2 \pi \sigma^{2}} \exp \left(-\frac{\left(x-x^{\prime}\right)^{2}+\left(y-y^{\prime}\right)^{2}}{2 \sigma^{2}}\right),
$$

where $\mathbf{x}:=(x, y), \mathbf{x}^{\prime}:=\left(x^{\prime}, y^{\prime}\right) \in \Omega$ and $\sigma$ is the model parameter characterizing the degree of accuracy and clearness of the image in the system.

The two-dimensional integral equation (2.1) with the kernel (2.3) may be written as a system of two univariate integral equations due to the symmetry of the kernel. That is, equation (2.1) is equivalent to the system

$$
\begin{aligned}
& \frac{1}{\sqrt{2 \pi} \sigma} \int_{0}^{1} \exp \left(-\frac{\left(y-y^{\prime}\right)^{2}}{2 \sigma^{2}}\right) u\left(x, y^{\prime}\right) d y^{\prime}=f(x, y), \\
& \frac{1}{\sqrt{2 \pi} \sigma} \int_{0}^{1} \exp \left(-\frac{\left(x-x^{\prime}\right)^{2}}{2 \sigma^{2}}\right) v\left(x^{\prime}, y^{\prime}\right) d x^{\prime}=u\left(x, y^{\prime}\right) .
\end{aligned}
$$

For a given observed blurred image $f$, we may obtain the original image by successively solving the above two one-dimensional integral equations. We first solve equation (2.4) for $u$ and then solve equation (2.5) for $v$. For this reason, in the remaining part of this paper, we shall focus on developing a collocation method for solving the onedimensional integral equation

$$
K v=h,
$$

where the integral operator $K$ is defined for $v \in X:=L^{\infty}(E)$ by

$$
(\mathcal{K} v)(x):=\int_{E} K(x, y) v(y) d y, \quad x \in E,
$$

with 


$$
K(x, y):=\frac{1}{\sqrt{2 \pi} \sigma} \exp \left(-\frac{(x-y)^{2}}{2 \sigma^{2}}\right), \quad x, y \in E,
$$

and $h \in X$ is a given function.

Integral equation (2.6) is ill-posed in the sense that its solution does not continuously depend on the given data $h$ due to the compactness of the integral operator $\mathcal{K}$ on $X$. Therefore, we need to use a regularization method to find its approximate solution. In this paper, we will adapt the Tikhonov regularization method. The Tikhonov regularization solution $v_{\lambda}$ of (2.6) is obtained by solving the second kind integral equation

$$
\left(\lambda \mathcal{I}+\mathcal{K}^{*} \mathcal{K}\right) v_{\lambda}=\mathcal{K}^{*} h,
$$

where $\mathcal{I}$ denotes the identity operator on $X, \mathcal{K}^{*}$ denotes the adjoint operator of $\mathcal{K}$ and $\lambda$ is a regularization parameter. Actually, due to measurement errors, the observed data is a noisy one, $h^{\delta}$ with

$$
\left\|h-h^{\delta}\right\|_{\infty} \leq \delta
$$

for a noise level $\delta$. We shall denote by $v_{\lambda}^{\delta}$ the solution of equation (2.9) with $h$ being replaced by $h^{\delta}$, and we rewrite equation (2.9) as

$$
\left(\lambda \mathcal{I}+\mathcal{K}^{*} \mathcal{K}\right) v_{\lambda}^{\delta}=\mathcal{K}^{*} h^{\delta} .
$$

The operator $\lambda \mathcal{I}+\mathcal{K}^{*} \mathcal{K}$ is strictly coercive, and thus, for any positive number $\lambda$, it has a bounded inverse [16]. Being a convolution-type operator, $K$ is self-adjoint, that is, $\mathcal{K}^{*}=\mathcal{K}$. Hence, equation (2.11) may be rewritten as

$$
\left(\lambda \mathcal{I}+\mathcal{K}^{2}\right) v_{\lambda}^{\delta}=\mathcal{K} h^{\delta} .
$$

Note that the integral operator $\mathcal{K}^{2}$ has the kernel

$$
\bar{K}(x, y):=\int_{E} K(x, z) K(z, y) d z, \quad x, y \in E .
$$

Clearly, from the above expression of the kernel of the integral operator $\mathcal{K}^{2}$, evaluation of $\left(\mathcal{K}^{2} v_{\lambda}^{\delta}\right)(x)$ requires computing a double integral, which is computationally expensive.

We shall develop an efficient collocation method for solving equation (2.12). A difficulty in the numerical solution of this equation is the involvement of the integral operator $\mathcal{K}^{2}$ in the equation. The 
collocation method applied directly to solving equation (2.12) leads to a linear system whose coefficient matrix has the entries involving $\left(\mathcal{K}^{2} v_{\lambda}^{\delta}\right)(x)$ for $x \in E$. Since it is computationally expensive to compute $\left(\mathcal{K}^{2} v_{\lambda}^{\delta}\right)(x)$ for $x \in E$, avoiding evaluating the double integrals is highly desirable. To this end, following an idea used in [3], we observe that equation (2.12) is equivalent to the following equation

$$
\mathcal{K}\left(\mathcal{K} v_{\lambda}^{\delta}-h^{\delta}\right)+\lambda v_{\lambda}^{\delta}=0 .
$$

By letting $\sqrt{\lambda} x_{\lambda}^{\delta}:=\mathcal{K} v_{\lambda}^{\delta}-h^{\delta}$, we write equation (2.13) as a system of equations

$$
\left\{\begin{array}{l}
\mathcal{K} x_{\lambda}^{\delta}+\sqrt{\lambda} v_{\lambda}^{\delta}=0, \\
\mathcal{K} v_{\lambda}^{\delta}-\sqrt{\lambda} x_{\lambda}^{\delta}=h^{\delta} .
\end{array}\right.
$$

Instead of solving equation (2.12) directly, we shall solve the system (2.14). The collocation method applied to system (2.14) leads to a linear system whose coefficient matrix has the entries involving $\left(\mathcal{K} v_{\lambda}^{\delta}\right)(x)$ for $x \in E$. Hence, the coefficient matrix of the linear system of the integral equation system involves only single integrals. Efficient methods can be developed for the integral equation system (2.14).

3. Multiscale collocation methods. This section is devoted to describing a multiscale collocation method for solving the integral equation system (2.14). The discretization of system (2.14) leads to a linear system having a dense coefficient matrix. We shall adapt the multiscale analysis presented in $[4,6,7,18,19]$ and the references cited therein to develop an efficient multiscale collocation method for solving system (2.14).

The multiscale analysis suitable for developing collocation methods for solving system (2.14) provides a multiscale basis of a subspace $V$ (which contains the continuous function space $C(E)$ ) of $X$ and a set of multiscale collocation functionals. The multiscale basis will be used to approximate the solution of system (2.14), and the set of functionals will be used to set up the collocation method.

We describe below the basis and the set of functionals. Specifically, we shall choose the solution space of the integral equation to be a space of piecewise polynomials on multiscale partitions. Following $[\mathbf{1 8}, \mathbf{1 9}]$, we construct a multiscale basis for the piecewise polynomial space. Let 
$N$ denote the set of all positive integers and $N_{0}:=N \cup\{0\}$. For $n \in N$, we let $Z_{n}:=\{0,1, \ldots, n-1\}$ and $Z_{\mu}^{n}:=Z_{\mu} \times \cdots \times Z_{\mu}$ (n times). For a fixed positive integer $\mu$ with $\mu>1$, we define a set $\Phi:=\left\{\varphi_{\epsilon}: \epsilon \in Z_{\mu}\right\}$ of contractive mappings on $E$ by

$$
\varphi_{\epsilon}(t):=\frac{\epsilon+t}{\mu}, \quad t \in E, \epsilon \in Z_{\mu} .
$$

Clearly, we have that

$$
E=\bigcup_{\epsilon \in Z_{\mu}} \varphi_{\epsilon}(E)
$$

The contractive mappings $\varphi_{\epsilon}$ will be used to form a sequence of multiscale partitions of $E$ on which the multiscale basis and the corresponding collocation functionals will be defined. Specifically, associated with these mappings, we define linear operators $\mathcal{T}_{\epsilon}: X \rightarrow X$ for $v \in X$ by

$$
\left(\mathcal{T}_{\epsilon} v\right)(\cdot):=\mu^{1 / 2}\left(v \circ \varphi_{\epsilon}^{-1}\right)(t) \chi_{\varphi_{\varepsilon}(E)}(t), \quad t \in E,
$$

where $\chi_{J}$ is the characteristic function of set $J \subset E$. We let $X_{0}:=\prod_{k}$ be the space of all polynomials of degree $\leq k-1$. With the given subspace $X_{0}$ and linear operators $\mathcal{T}_{\epsilon}$, we generate recursively a sequence of multiscale piecewise polynomial spaces $X_{n}$ by

$$
X_{n}:=\bigoplus_{\epsilon \in Z_{\mu}} \mathcal{T}_{\epsilon}\left(X_{n-1}\right), \quad n \in N .
$$

Note that $X_{n}$ is a space of piecewise polynomials of degree $k-1$ and $X_{n} \subset X_{n+1}$. We let $W_{n+1}$ denote the orthogonal complement of $X_{n}$ in $X_{n+1}$, that is, $X_{n+1}=X_{n} \oplus^{\perp} W_{n+1}$. With the convention that $W_{0}=X_{0}$, we have that $X_{n}=W_{0} \oplus^{\perp} W_{1} \oplus^{\perp} \cdots \oplus^{\perp} W_{n}$, and the spaces $W_{j}, j \in Z_{n+1}$, form a multiscale analysis of the space $X_{n}$. Moreover, when $W_{1}$ is available, we have for $n \in N$ that

$$
W_{n+1}=\oplus_{\epsilon \in Z_{\mu}} \mathcal{T}_{\epsilon}\left(W_{n}\right) .
$$

For $j \in N$, we let $w(j):=\operatorname{dim} W_{j}$, and it is easy to verify that $w(1)=r:=(\mu-1) k$ and $w(i)=\mu^{i-1} r$. We may choose the $k$ orthonormal polynomials $\left\{w_{0_{j}}: j \in Z_{k}\right\}$ on $E$ as a basis of $X_{0}$ and construct an orthonormal basis $\left\{w_{1 j}: j \in Z_{w(1)}\right\}$ of $W_{1}$ via the GramSchmidt process.

The multiscale basis $\left\{w_{i j}: j \in Z_{w(i)}\right\}$ of $W_{i}$ can then be constructed from that of $W_{i-1}$ by applying the operators $\mathcal{T}_{\epsilon}$. The basis functions 
$w_{i j}$ may be expressed in terms of consecutive applications of operators $\mathcal{T}_{\epsilon}$ to basis functions of $W_{1}$. To see this, note that each $j \in Z_{\mu^{i}}$ can be uniquely written as $j=\mu^{i-1} s_{0}+\cdots+\mu s_{i-2}+s_{i-1}$ for some $\mathbf{s}=\left[s_{0}, s_{1}, \ldots, s_{i-1}\right] \in Z_{\mu}^{i}$. Hence, we write $j=\mu(\mathbf{s})$. For $\mathbf{s}=\left[s_{0}, s_{1}, \ldots, s_{i-2}\right] \in Z_{\mu}^{i-1}$, we let $\mathcal{T}_{\mathbf{s}}:=\mathcal{T}_{s_{0}} \circ \mathcal{T}_{s_{1}} \circ \cdots \circ \mathcal{T}_{s_{i-2}}$. Then, for $j \in Z_{w(i)}, j=\mu(\mathbf{s}) r+\ell$ with $i>1, \mathbf{s} \in Z_{\mu}^{i-1}$ and $\ell \in Z_{r}$, we have that $w_{i j}=\mathcal{T}_{\mathbf{s}} w_{1 \ell}$. By defining the index set $U_{n}:=\left\{(i, j): i \in Z_{n+1}, j \in Z_{w(i)}\right\}$, we conclude that $\left\{w_{i j}:(i, j) \in U_{n}\right\}$ forms a multiscale orthonormal basis for $X_{n}$.

We now identify the support of $w_{i j}$. For each $i \in N$ with $i>1$ and, for $s \in Z_{\mu}^{i-1}$, we let $\Phi_{s}:=\varphi_{s_{0}} \circ \varphi_{s_{1}} \circ \cdots \circ \varphi_{s_{i-2}}$ and define $E_{i \mathbf{s}}:=\Phi_{s}(E)$. Clearly, we have that

$$
\bigcup_{\mathbf{s} \in Z_{\mu}^{i-1}} E_{i, \mathbf{s}}=E
$$

and

$$
\operatorname{meas}\left(E_{i, \mathbf{s}} \cap E_{i, \mathbf{s}^{\prime}}\right)=0 \text { for } \mathbf{s} \neq \mathbf{s} \text {. }
$$

Moreover, letting $S_{i j}:=E_{i, \mathbf{s}}$, for $i>1$ and $j=\mu(\mathbf{s}) r+\ell$ with $s \in Z_{\mu}^{i-1}$, $l \in Z_{r}$, we define $E_{0, j}:=E, j \in Z_{k}$ and $E_{1, j}:=E, j \in Z_{r}$. Clearly, the support of $w_{i j}$ is contained in $S_{i j}$.

Next, we turn to describing the sequence of the multiscale collocation functionals. Corresponding to each basis function $w_{i j}$, we have a collocation functional $\ell_{i j}$, which is a sum of point evaluation functions at a fixed number of points in $S_{i j}$. Specifically, following [4], we choose a finite set $G_{0}:=\left\{t_{i}: j \in Z_{k}\right\}$ of distinct points in $E$, which is refinable relative to the mappings $\Phi$ in the sense that

$$
\Phi\left(G_{0}\right):=\bigcup_{\epsilon \in Z_{\mu}} \varphi_{\epsilon}\left(G_{0}\right) \supset G_{0} .
$$

We let $G_{1}:=\Phi\left(G_{0}\right)$ and $V_{1}:=G_{1} \backslash G_{0}=\left\{t_{k+j}: j \in Z_{r}\right\}$. For $j \in Z_{k}$, we define $\ell_{0 j}:=\delta_{t_{j}}$. For $j^{\prime} \in Z_{r}$ and $q:=k+r$, we find the vector $\left[c_{j s}: s \in Z_{q}\right]$ such that $\ell_{1 j^{\prime}}:=\sum_{s \in Z_{q}} c_{j s} \delta_{t_{s}}, j \in Z_{r}$, satisfies the equations $\left\langle\ell_{1 j^{\prime}}, w_{0 j}\right\rangle=0$, for $j \in Z_{k}$, and $\left\langle\ell_{1 j^{\prime}}, w_{1 j}\right\rangle=\delta_{j^{\prime} j}$, for $j \in Z_{r}$. To generate the multiscale collocation functionals, we introduce for any $s \in Z_{\mu}$ a linear operator $\mathcal{L}_{s}: X^{*} \rightarrow X^{*}$ defined by the equation $\left\langle\mathcal{L}_{s} \ell, v\right\rangle=\left\langle\ell, v \circ \varphi_{s}\right\rangle, v \in X, \ell \in X^{*}$, where $X^{*}$ denotes the dual 
space of $X$. Moreover, for $\mathbf{s}:=\left[s_{0}, s_{1}, \ldots, s_{i-1}\right] \in Z_{\mu}^{i}$, we define the composition operator as $\mathcal{L}_{s}:=\mathcal{L}_{s_{0}} \circ \cdots \circ \mathcal{L}_{s_{i-1}}$. For $i>1, j=\mu(s) r+l$ with $\mathbf{s} \in Z_{\mu}^{i-1}, l \in Z_{r}$, we define $\ell_{i j}:=\mathcal{L}_{s} \ell_{1 l}$ and observe that

$$
\left\langle\ell_{i j}, v\right\rangle=\left\langle\ell_{1 l}, v \circ \varphi_{\mathbf{s}}\right\rangle=\sum_{s \in Z_{q}} c_{l s} v\left(\varphi_{s}\left(t_{s}\right)\right) .
$$

Clearly, the "support" $\widehat{S}_{i j}$ of $\ell_{i j}$ is also contained in $S_{i j}$.

We next summarize several useful properties of the basis functions and the collocation functionals that we constructed above. To this end, we let $\mathcal{P}_{n}$ denote the projection from $X$ onto $X_{n}$ defined by the requirement that

$$
\left\langle\ell_{i j}, \mathcal{P}_{n} x\right\rangle=\left\langle\ell_{i j}, x\right\rangle, \quad(i, j) \in U_{n} .
$$

We shall use $c$ to denote a universal constant which can be distinct at different occurrences in the remaining sections.

Proposition 3.1. The following properties hold:

(i) For any $n \in N_{0},\left\langle\ell_{i^{\prime} j^{\prime}}, w_{i j}\right\rangle=\delta_{i i^{\prime}} \delta_{j j^{\prime}},(i, j),\left(i^{\prime}, j^{\prime}\right) \in U n, i \leq i^{\prime}$.

(ii) For $p \in \prod_{k},\left\langle\ell_{i j}, p\right\rangle=0,\left\langle w_{i j}, p\right\rangle=0,(i, j) \in U_{n}$.

(iii) There exists a positive constant $\theta_{0}$ such that, for all $(i, j) \in U_{n}$, $\left.\| \ell_{i j}\right\}+\left\|w_{i j}\right\|_{\infty} \leq \theta_{0}$ holds.

(iv) If $\operatorname{dim} X_{i}=s(i)$ and $\max \left\{\right.$ meas $\left.\left(E_{i, \mathbf{s}}\right): \mathbf{s} \in Z_{\mu}^{i-1}\right\}=d_{i}$, then $s(i) \sim \mu^{i}, w(i) \sim \mu^{i}$ and $d_{i} \sim \mu^{-i}$.

(v) The operators $\mathcal{P}_{n}$ are well defined and converge pointwise to the identity operator $\mathcal{I} \in X$ as $n \rightarrow \infty$, that is, for each $x \in X$, $\lim _{n \rightarrow \infty}\left\|\mathcal{P}_{n} x-x\right\|=0$ holds.

(vi) There exists a positive constant $c$ such that, for all $u \in W^{k, \infty}$, $\operatorname{dist}\left(u, X_{n}\right) \leq c \mu^{-k n}\|u\|_{k, \infty}$.

(vii) There exist positive constants $\theta_{1}$ and $\theta_{2}$ such that, for all $n \in N_{0}$ and $v \in X_{n}$ having the form $v=\sum_{(i, j) \in U_{n}} v_{i j} w_{i j}$, the following inequality holds

$$
\theta_{1}\|v\|_{\infty} \leq\|v\|_{\infty} \leq \theta_{2}(n+1)\left\|\mathbf{E}_{n} \mathbf{v}\right\|_{\infty},
$$

where $\mathbf{v}:=\left[v_{i j}:(i, j) \in U_{n}\right]^{T} s$.

The proof of these properties may be found in [7]. 
We now describe the multiscale collocation method for solving system (2.14) of integral equations. For $n \in N$, let $\mathcal{K}_{n}:=\left.\mathcal{P}_{n} \mathcal{K}\right|_{X_{n}}$. The multiscale collocation method for system (2.14) seeks vectors $\mathbf{v}_{\lambda, n}^{\delta}:=\left[v_{i j}^{\lambda, n}:(i, j) \in U_{n}\right]^{T}$ and $\mathbf{x}_{\lambda, n}^{\delta}:=\left[x_{i j}^{\lambda, n}:(i, j) \in U_{n}\right]^{T}$ such that

$$
v_{\lambda, n}^{\delta}:=\sum_{(i, j) \in U_{n}} v_{i j}^{\lambda, n} w_{i j}
$$

and

$$
x_{\lambda, n}^{\delta}:=\sum_{(i, j) \in U_{n}} x_{i j}^{\lambda, n} w_{i j}
$$

are the solution of

$$
\left\{\begin{array}{l}
\mathcal{K}_{n} x_{\lambda, n}^{\delta}+\sqrt{\lambda} v_{\lambda, n}^{\delta}=0 \\
\mathcal{K}_{n} v_{\lambda, n}^{\delta}-\sqrt{\lambda} x_{\lambda, n}^{\delta}=\mathcal{P}_{n} h^{\delta}
\end{array}\right.
$$

By introducing

$$
\begin{aligned}
\mathbf{E}_{n} & :=\left[\left\langle\ell_{i^{\prime} j^{\prime}}, w_{i j}\right\rangle:(i, j),\left(i^{\prime}, j^{\prime}\right) \in U_{n}\right], \\
\mathbf{K}_{n} & :=\left[\left\langle\ell_{i^{\prime} j^{\prime}}, \mathcal{K} w_{i j}\right\rangle:(i, j),\left(i^{\prime}, j^{\prime}\right) \in U_{n}\right]
\end{aligned}
$$

and

$$
\left.\mathbf{h}_{n}^{\delta}=\left\langle\ell_{i^{\prime} j^{\prime}}, h^{\delta}\right\rangle:\left(i^{\prime}, j^{\prime}\right) \in U_{n}\right]
$$

equation (3.3) has the matrix form

$$
\left[\begin{array}{cc}
\mathbf{K}_{n}, & -\sqrt{\lambda} \mathbf{E}_{n} \\
\sqrt{\lambda} \mathbf{E}_{n}, & \mathbf{K}_{n}
\end{array}\right]\left[\begin{array}{c}
\mathbf{v}_{\lambda, n}^{\delta} \\
\mathbf{x}_{\lambda, n}^{\delta}
\end{array}\right]=\left[\begin{array}{c}
\mathbf{h}_{n}^{\delta} \\
\mathbf{0}
\end{array}\right] .
$$

Upon solving system (3.4), we obtain an approximate solution of the system (2.14). To close this section, we compare the solution of system (2.14) as an approximation of the reconstructed image with that of the discrete model. When the basis functions are chosen as the piecewise constant approximation, the solution of system (2.14) is in fact the same as the solution of the discrete model. An advantage of using the continuous model is that it allows us to use piecewise polynomial approximations of a higher order. They will give us more accurate approximations of the reconstructed image. Higher order piecewise polynomial approximation is particularly suitable for image 
enlargement, since the basis functions chosen are defined on the entire image domain. Image enlargement for an image generated from a discrete model requires certain interpolation, which normally gives poor quality. For numerical comparison, the reader is referred to [16]. However, the use of a high order piecewise polynomial approximation will lead to higher computational complexity. This is a main issue that we shall address in the next section.

4. Generation of the compressed matrix. In this section, we propose a matrix compression strategy for the coefficient matrix of the linear system (3.4) and develop a quadrature scheme for computing the remaining nonzero entries of the resulting compressed matrix.

We first consider compressing the coefficient matrix of the linear system (3.4), which is formed by two matrices $\mathbf{E}_{n}$ and $\mathbf{K}_{n}$. Note that, according to property (i) in Proposition 3.1, matrix $\mathbf{E}_{n}$ is a block upper triangular matrix with the identity matrices in the diagonal blocks, which is already sparse. However, matrix $\mathbf{K}_{n}$ is a dense matrix with each of its entries an integral involving the Gaussian kernel. Although the Gaussian kernel is smooth, when its variance is small, the kernel behaves like a singular function, and computing integrals involving the kernel needs special care. Generating such a matrix requires much computational cost when the size of the matrix is large. In order to reduce the computational cost and yet retain the order of accuracy of the approximate solution, we propose to truncate the matrix $\mathbf{K}_{n}$ according to the properties of the Gaussian kernel.

We denote by $K_{i^{\prime} j^{\prime}, i j}$ the entries of matrix $\mathbf{K}_{n}$, that is, $K_{i^{\prime} j^{\prime}, i j}:=$ $\left\langle\ell i^{\prime} j^{\prime}, \mathcal{K} w_{i j}\right\rangle$. We partition $\mathbf{K}_{n}$ as a block matrix

$$
\mathbf{K}_{n}=\left[\mathbf{K}_{i^{\prime} i}: i, i^{\prime} \in Z_{n+1}\right],
$$

where

$$
\mathbf{K}_{i^{\prime} i}:=\left[K_{i^{\prime} j^{\prime}, i j}: j^{\prime} \in Z_{\omega\left(i^{\prime}\right)}, j \in Z_{\omega(i)}\right] .
$$

We use dist $(Y, Z):=\min \{|y-z|: y \in Y, z \in Z\}$ to denote the distance between two compact sets $Y$ and $Z$ of $R$. For each $i, i^{\prime} \in Z_{n+1}$, we choose a truncation parameter $\delta_{i^{\prime} i}$ and introduce the matrix $\widehat{K}_{i^{\prime} i}:=$ 
$\left[\widehat{K}_{i^{\prime} j^{\prime}, i j}: j^{\prime} \in Z_{\omega\left(i^{\prime}\right)}, j \in Z_{\omega(i)}\right]$, where

$$
\widehat{K}_{i^{\prime} j^{\prime}, i j}:= \begin{cases}K_{i^{\prime} j^{\prime}, i j} & \text { if } \operatorname{dist}\left(S_{i^{\prime} j^{\prime}}, S_{i j}\right) \leq \sigma \delta_{i^{\prime} i}, \\ 0 & \text { otherwise. }\end{cases}
$$

We then define the compressed matrix $\widehat{\mathbf{K}}_{n}$ by letting $\widehat{\mathbf{K}}_{n}:=\left[\widehat{\mathbf{K}}_{i^{\prime} i}\right.$ : $\left.i^{\prime}, i \in Z_{n+1}\right]$ and replace the matrix $\widehat{\mathbf{K}}_{n}$ in (3.4) by $\widehat{\mathbf{K}}_{n}$. This gives rise to the compressed linear system

$$
\left[\begin{array}{cc}
\widehat{\mathbf{K}}_{n} & -\sqrt{\lambda} \mathbf{E}_{n} \\
\sqrt{\lambda} \mathbf{E}_{n} & \widehat{\mathbf{K}}_{n}
\end{array}\right]\left[\begin{array}{c}
\widehat{\mathbf{v}}_{n, \lambda}^{\delta} \\
\widehat{\mathbf{x}}_{n, \lambda}^{\delta}
\end{array}\right]=\left[\begin{array}{c}
\mathbf{h}_{n}^{\delta} \\
\mathbf{0}
\end{array}\right] .
$$

We solve the linear system (4.2) for vectors $\widehat{\mathbf{v}}_{n, \lambda}^{\delta}$ and $\widehat{x}_{n, \lambda}^{\delta}$, which provide an approximation of $\mathbf{v}_{n, \lambda}^{\delta}$ and $\mathbf{x}_{n, \lambda}^{\delta}$, respectively.

In the truncation scheme described above, there is a parameter $\delta_{i^{\prime} i}$ which may depend on $i, i^{\prime}$ needing to be determined. We choose it in a way similar to the Galerkin method described in [16]. Specifically, we let

$$
g(x):=\frac{1}{\sqrt{2 \pi}} \exp \left(-\frac{x^{2}}{2}\right), \quad x \in R
$$

denote the normalized Gaussian function, and define

$$
G_{2 k}(t):=\int_{t}^{\infty}\left|g^{(2 k)}(\xi)\right| d \xi, \quad t \in[0, \infty) .
$$

For two given numbers $\alpha$ and $\alpha^{\prime}$, we let

$$
\epsilon_{i^{\prime} i}:=a^{\eta} \mu^{-\eta\left(-n+\alpha(n-i)+\alpha^{\prime}\left(n-i^{\prime}\right)\right)} \sigma^{2 k},
$$

where $\eta:=2 k 1$. If $\epsilon_{i^{\prime} i}<G_{2 k}(0)$, we choose $\delta_{i^{\prime} i}$ such that

$$
G_{2 k}\left(\delta_{i^{\prime} i}\right)=\epsilon_{i^{\prime} i},
$$

and otherwise, we choose

$$
\delta_{i^{\prime} i}:=0 .
$$

The nonlinear equation (4.5) may be solved numerically with the method described in [16].

We next estimate the number of nonzero entries in matrix $\widehat{\mathbf{K}}_{n}$. For a matrix $K$, we denote by $\mathcal{N}(\mathbf{K})$ the number of nonzero entries in $\mathbf{K}$. 
Theorem 4.1. If $\alpha$ and $\alpha^{\prime}$ are real numbers not larger than one, and the truncation parameters $\delta_{i^{\prime} i}$ are chosen as in (4.5) and (4.6), then there exists a positive constant $c$ such that, for all $\sigma>0$,

$$
N\left(\widehat{\mathbf{K}}_{n}\right) \leq c s(n) \log ^{\tau} s(n),
$$

where $\tau=1$, except for $\alpha=\alpha^{\prime}=1$, in which case $\tau=2$.

Proof. We first estimate the number $\mathcal{N}\left(\widehat{\mathbf{K}}_{i^{\prime} i}\right)$. For fixed $i, i^{\prime}$ and $j^{\prime}$, if $\widehat{K}_{i^{\prime} i} \neq 0$, then we have that $\operatorname{dist}\left(S_{i^{\prime} j^{\prime}}, S_{i j}\right) \leq \sigma \delta_{i^{\prime} i}$. This ensures that

$$
S_{i j} \subseteq S\left(i, i^{\prime}\right):=\left\{x: x \in R,\left|x-x_{0}\right| \leq d_{i}+d_{i^{\prime}}+\sigma \delta_{i^{\prime} i}\right\},
$$

where $x_{0}$ is an arbitrary point in the set $S_{i^{\prime} j^{\prime}}$. Let $\mathcal{N}_{i, i^{\prime} j^{\prime}}$ denote the number of the sets contained in $S\left(i, i^{\prime}\right)$. Using Proposition 3.1 (iv), we conclude that there exists a positive constant $c$ such that

$$
\mathcal{N}_{i, i^{\prime} j^{\prime}} \leq \frac{\operatorname{meas}\left(S\left(i, i^{\prime}\right)\right)}{\min \left\{\operatorname{meas}\left(S_{i j}\right): S_{i j} \subseteq S(i, i)\right\}} \leq c \mu^{i}\left(d_{i}+d_{i^{\prime}}+\sigma \delta_{i^{\prime} i}\right) .
$$

See [20] for more details. Note that the number of functions $w_{i j}$ having support contained in $S_{i j}$ is bounded by $r:=\omega(1)$. Appealing to Proposition 3.1 (iv), we conclude that there exists a positive constant $c$ such that

$$
\mathcal{N}\left(\widehat{\mathbf{K}}_{i^{\prime} i}\right) \leq r \sum_{j^{\prime} \in Z_{\omega\left(i^{\prime}\right)}} \mathcal{N}_{i, i^{\prime} j^{\prime}} \leq c \mu^{i+i^{\prime}}\left(d_{i}+d_{i^{\prime}}+\sigma \delta_{i^{\prime} i}\right), \quad i, i^{\prime} \in Z_{n+1} .
$$

It remains to estimate $\mathcal{N}\left(\widehat{\mathbf{K}}_{n}\right)$. It follows from (4.8) that

$$
\mathcal{N}\left(\widehat{\mathbf{K}}_{n}\right) \leq c\left(\mathcal{N}_{1}+\mathcal{N}_{2}\right),
$$

where

$$
\begin{aligned}
\mathcal{N}_{1} & :=\sum_{i \in Z_{n+1}} \sum_{i^{\prime} \in Z_{n+1}} \mu^{i+i^{\prime}}\left(d_{i}+d_{i^{\prime}}\right), \\
\mathcal{N}_{2} & :=\sum_{i \in Z_{n+1}} \sum_{i^{\prime} \in Z_{n+1}} \mu^{i+i^{\prime}} \sigma \delta_{i^{\prime} i} .
\end{aligned}
$$

Since $d_{i} \sim \mu^{-i}$ and $d_{i^{\prime}} \sim \mu^{-i^{\prime}}$, we obtain that

$$
\mathcal{N}_{1} \leq c(n+1) \mu^{n} \text {. }
$$


We next estimate $\mathcal{N}_{2}$. Noting that $\lim _{\xi \rightarrow \infty}\left|g^{2 k}(\xi) \xi^{2 k}\right| \leq c$, which implies that

$$
\frac{c}{|\xi|^{2 k}} \geq\left|g^{(2 k)}(\xi)\right| .
$$

Integrating both sides of the above inequality over $\left[\delta_{i^{\prime} i}, \infty\right)$ yields

$$
\int_{\delta_{i^{\prime} i}}^{\infty} \frac{c}{|\xi|^{2 k}} d \xi \geq \int_{\delta_{i^{\prime} i}}^{\infty}\left|g^{(2 k)}(\xi)\right| d \xi
$$

If $\delta_{i^{\prime} i} \neq 0$, computing the integral on the left-hand side and using (4.5), we have that

$$
\frac{c}{2 k-1} \delta_{i^{\prime} i}^{-(2 k-1)} \geq a^{-\eta} \mu^{-\eta\left(-n+\alpha(n-i)+\alpha^{\prime}\left(n-i^{\prime}\right)\right)} \sigma^{2 k},
$$

which ensures that

$$
\sigma \delta_{i^{\prime} i} \leq c a \frac{1}{\sigma^{1 / 2 k-1}} \mu^{-n}+\alpha(n-i)+\alpha^{\prime}\left(n-i^{\prime}\right) .
$$

Using (4.11), we have that

$$
\mathcal{N}_{2} \leq c a \frac{1}{\sigma^{1 / 2 k-1}} \mu^{n} \sum_{i \in Z_{n+1}} \mu^{(\alpha-1)(n-i)} \sum_{i^{\prime} \in Z_{n+1}} \mu^{\left(\alpha^{\prime}-1\right)\left(n-i^{\prime}\right)}=c \mu^{n} n^{\tau},
$$

which, together with (4.9) and (4.10) proves the desired result of this theorem.

We next turn to discussing computation of the nonzero entries of the matrix $\widehat{\mathbf{K}}_{n}$. These entries are integrals whose integrands involve products of the Gaussian kernel and piecewise polynomials. When the variance of the Gaussian kernel is small, the kernel behaves like a singular function, although it is smooth. Hence, the integration of these functions requires special treatment. The nonzero entries $\widehat{K}_{i^{\prime} j^{\prime}, i j}$ of matrix $\widehat{\mathbf{K}}_{n}$ needing to be computed have the form

$$
\widehat{K}_{i^{\prime} j^{\prime}, i j}=\sum_{x \in \hat{S}_{i^{\prime} j^{\prime}}} c_{x} \int_{S_{i j}} h_{i j}(x, y) d y
$$

where $h_{i j}(x, y):=K(x, y) w_{i j}(y)$. Recalling that the Gaussian kernel $K$ is not an elementary function, the above integrals cannot be computed exactly. As a result, we have to resort to numerical methods for evaluating $\widehat{K}_{i^{\prime} j^{\prime}, i j}$. We shall develop a numerical quadrature scheme 
for computing the integrals

$$
I\left(h_{i j}\right):=\int_{S_{i j}} h_{i j}(x, y) d y,
$$

so that the quadrature error will not ruin the overall convergence order of the resulting approximate solution. To this end, following similar ideas proposed in $[\mathbf{8}, \mathbf{1 5}, \mathbf{1 6}]$, we shall use an appropriate partition of $S_{i j}$ and apply the Gauss-Legendre quadrature formula to the integrals on subintervals.

Let $\epsilon>0$ be a given fixed number, and let $m$ be a positive integer. We choose $\beta$ to satisfy that

$$
1-\beta<\sigma \beta^{1+1 / 2 \epsilon} .
$$

Let $t_{0}:=0$ and $t_{\iota}:=\beta^{m-\iota}$ for $\iota \in Z_{m+1} \backslash\{0\}$, and choose a sequence of nonnegative integers $k_{\iota}:=[\epsilon \ell]+1$ for $\iota \in Z_{m}$, where $[x]$ is the largest integer less than or equal to $x$. For a point $x$, we pick two collections of nodes $\pi_{t}^{r}:=\left\{t_{\iota}^{r}:=x+t_{\iota}: \iota \in Z_{m+1} \backslash\{0\}\right\}$ and $\pi_{t}^{l}:=\left\{t_{\iota}^{l}:=x-t_{\iota}: \iota \in Z_{m+1} \backslash\{0\}\right\}$. Let $\pi\left(h_{i j}\right)$ be a set $\pi\left(h_{i j}\right):=\left\{x_{\ell}: \ell-1 \in Z_{m^{\prime}-1}\right\}$ such that $h_{i j} \in C^{(k-1)}\left(E \backslash \pi\left(h_{i j}\right)\right)$ and $\left[q^{\prime}, q^{\prime \prime}\right]:=\operatorname{supp}\left(h_{i j}\right)$. We arrange the elements of the set

$$
\left(\pi\left(h_{i j}\right) \cup \pi_{t}^{r} \cup \pi_{t}^{l} \cup\left\{q^{\prime}, q^{\prime \prime}\right\}\right) \cap \operatorname{supp}\left(h_{i j}\right)
$$

in the increasing order and write them as a new sequence

$$
q^{\prime}=q_{0}<q_{1}<\cdots<q_{m^{\prime \prime}}=q^{\prime \prime},
$$

where $m^{\prime \prime} \leq 2 m+m^{\prime}+1$. We define the partition $\prod\left(h_{i j}\right):=\left\{Q_{\alpha}:=\right.$ $\left.\left[q_{\alpha}, q_{\alpha+1}\right): \alpha \in Z_{m^{\prime \prime}}\right\}$. For each interval $\left[q_{\alpha}, q_{\alpha+1}\right]$, there exists an $\iota \in Z_{m}$ such that $\left[q_{\alpha}, q_{\alpha+1}\right] \subseteq\left[t_{\iota}^{r}, t_{\iota+1}^{r}\right]$ or $\left[q_{\alpha}, q_{\alpha+1}\right] \subseteq\left[t_{\iota+1}^{l}, t_{l}^{l}\right]$. We approximate the integral $I\left(h_{i j}\right)$ by computing the integral on each interval $\left[q_{\alpha}, q_{\alpha+1}\right]$ using the Gauss-Legendre quadrature having precision of order $2 k_{\iota}-1$.

The integral of $h_{i j}$ on $\left[q_{\alpha}, q_{\alpha+1}\right]$ is computed approximately. Let $g_{k_{\iota}}$ denote the Legendre polynomial of degree $k_{\iota}$ on $E$, let $\left\{\tau_{\ell}: \ell \in Z_{k_{\ell}}\right\}$ denote its $k_{\iota}$ zeros with the order $0<\tau_{0}<\cdots<\tau_{k_{\iota}-1}<1$, and let $\tau_{\alpha}^{\ell}:=q_{\alpha}+\left(q_{\alpha+1}-q_{\alpha}\right)_{\ell}, \ell \in Z_{k_{\iota}}$. We then construct a piecewise polynomial $S_{\alpha}\left(h_{i j}\right)$ of order $k_{\iota}$, which interpolates $h_{i j}$ at points $\tau_{\alpha}^{\ell}$, $\ell \in Z_{k_{\iota}}$ on $\left[q_{\alpha}, q_{\alpha+1}\right]$ and is equal to zero outside $\left[q_{\alpha}, q_{\alpha+1}\right]$. We use 
$\sum_{\alpha \in Z_{m^{\prime \prime}}} I\left(S_{\alpha}\left(h_{i j}\right)\right)$ to approximate $I\left(h_{i j}\right)$ and use

$$
\widetilde{K}_{i^{\prime} j^{\prime}, i j}:=\sum_{x \in \hat{S}_{i^{\prime} j^{\prime}}} c_{x} \sum_{\alpha \in Z_{m^{\prime \prime}}} I\left(S_{\alpha}\left(h_{i j}\right)\right)
$$

to approximate the nonzero entry $\widehat{K}_{i^{\prime} j^{\prime}, i j}$. We replace the nonzero entries $\widehat{K}_{i^{\prime} j^{\prime}, i j}$ of $\widehat{\mathbf{K}}_{i^{\prime} i}$ by $\widetilde{K}_{i^{\prime} j^{\prime}, i j}$ and denote by $\widetilde{\mathbf{K}}_{i^{\prime} i}$ the resulting matrix block, and we use these blocks to form the matrix $\widetilde{\mathbf{K}}_{n}$. In system (4.2), we replace the matrix $\widehat{\mathbf{K}}_{n}$ by $\widetilde{\mathbf{K}}_{n}$ to obtain the approximate system

$$
\left[\begin{array}{cc}
\widetilde{\mathbf{K}}_{n} & -\sqrt{\lambda} \mathbf{E}_{n} \\
\sqrt{\lambda} \mathbf{E}_{n} & \widetilde{\mathbf{K}}_{n}
\end{array}\right]\left[\begin{array}{c}
\widetilde{\mathbf{v}}_{n, \lambda}^{\delta} \\
\widetilde{\mathbf{x}}_{n, \lambda}^{\delta}
\end{array}\right]=\left[\begin{array}{c}
\mathbf{h}_{n}^{\delta} \\
\mathbf{0}
\end{array}\right],
$$

where $\widetilde{\mathbf{v}}_{\lambda, n}^{\delta}:=\left[\widetilde{v}_{i j}^{\lambda, \delta}:(i, j) \in U_{n}\right]$ and $\widetilde{\mathbf{x}}_{\lambda, n}^{\delta}:=\left[\widetilde{x}_{i j}^{\lambda, \delta}:(i, j) \in U_{n}\right]$. Defining $\widetilde{v}_{\lambda, n}^{\delta}:=\sum_{(i, j) \in U_{n}} \widetilde{v}_{i j}^{\lambda, \delta} w_{i j}$ and $\widetilde{x}_{\lambda, n}^{\delta}:=\sum_{(i, j) \in U_{n}} \widetilde{x}_{i j}^{\lambda, \delta} w_{i j}$, we observe that $\widetilde{v}_{\lambda, n}^{\delta}, \widetilde{x}_{\lambda, n}^{\delta} \in X_{n}$, and they are an approximate solution of system (2.14).

To close this section, we present an estimate of the computational complexity for generating the matrix $\widetilde{\mathbf{K}}_{n}$ in terms of the total number $\mathcal{M}_{n}$ of functional evaluations used in computing all nonzero entries of the matrix. We denote by $\mathcal{M}_{i^{\prime} i}$ the number of functional evaluations in computing the nonzero entries of block $\widetilde{\mathbf{K}}_{i^{\prime} i}$. Thus, $\mathcal{M}_{n}=\sum_{i^{\prime} i \in Z_{n+1}} \mathcal{M}_{i^{\prime} i}$.

Theorem 4.2. If $\delta_{i^{\prime} i}$ is chosen according to (4.5) and (4.6) with $\alpha \leq 1$ and $\alpha^{\prime} \leq 1$, then there exists a positive constant $c$ such that, for all $\sigma$ and $n \in Z_{N}$,

$$
\mathcal{M}_{n} \leq \operatorname{cs}(n) \log ^{3} s(n)
$$

Proof. For $i^{\prime}, i \in Z_{n+1}$, we let $\mathcal{M}_{i^{\prime} j^{\prime}, i}$ denote the number of functional evaluations used in computing the $j^{\prime}$ th row of the block $\widetilde{\mathbf{K}}_{i^{\prime} i}$. Recalling that the number of rows in the block is $w\left(i^{\prime}\right)$, we have that

$$
\mathcal{M}_{i^{\prime} i}=w\left(i^{\prime}\right) M_{i^{\prime} j^{\prime}, i}
$$

To estimate $\mathcal{M}_{i^{\prime} j^{\prime}, i}$, we let $\mathcal{M}(h)$ denote the number of functional 
evaluations used in computing $I(S(h))$. We then have that

$$
\mathcal{M}_{i^{\prime} j^{\prime}, i}=\sum_{j \in Z_{i^{\prime} j^{\prime}, i}} \mathcal{M}\left(h_{i j}\right)
$$

Associated with the geometric partition described earlier in this section, there is a unique $L_{i^{\prime} i} \in Z_{m^{\prime \prime}}$ such that

$$
t_{L_{i^{\prime} i}-1} \leq \min \left\{\sigma \delta_{i^{\prime} i}+d_{i}+d_{i^{\prime}}\right\}
$$

and

$$
t_{L_{i^{\prime} i}} \geq \min \left\{\sigma \delta_{i^{\prime} i}+d_{i}+d_{i^{\prime}}\right\} .
$$

By using the fact that $k_{\iota}=[\epsilon l]+1$, we have that

$$
\begin{aligned}
\sum_{j \in Z_{i^{\prime} j^{\prime}, i}} \mathcal{M}\left(h_{i j}\right) & \leq \sum_{\iota \in Z_{L_{i^{\prime} i}}} \sum_{\left[q_{\alpha}, q_{\alpha+1}\right] \subset\left(\left[t_{\iota}^{r}, t_{\iota+1}^{r}\right] \cup\left[t_{\iota}^{l}, t_{\iota+1}^{l}\right]\right)} k_{\iota} \\
& \leq\left(2 \mu^{i}\left(d_{i}+d_{i^{\prime}}+\sigma \delta_{i^{\prime} i}\right)+2 L_{i^{\prime} i}\right)\left(\epsilon L_{i^{\prime} i}+1\right) .
\end{aligned}
$$

We can see that $m^{\prime \prime} \leq c n$ for a constant $c$, independent of $i$ and $i^{\prime}$. Thus, we obtain that

$$
\mathcal{M}_{i^{\prime} j^{\prime}, i} \leq c_{1} \mu^{i} n\left(d_{i}+d_{i^{\prime}}+\sigma \delta_{i^{\prime} i}\right)+c_{2} n^{2} .
$$

By using (4.11), we further have that

$$
\mathcal{M}_{i^{\prime} i} \leq c_{1} n \mu^{i+i^{\prime}}\left(\mu^{-n+\alpha(n-i)+\alpha^{\prime}\left(n-i^{\prime}\right)}+\mu^{-i}+\mu^{-i^{\prime}}\right)+c_{2} \mu^{i^{\prime}} n^{2} .
$$

This leads to the estimate

$$
\begin{aligned}
\mathcal{M}_{n} & \leq c_{1} n \sum_{i^{\prime} \in Z_{n+1}} \sum_{i \in Z_{n+1}} \mu^{i^{\prime}+i}\left(\mu^{-n+\alpha(n-i)+\alpha^{\prime}\left(n-i^{\prime}\right)}+\mu^{-i}+\mu^{-i^{\prime}}\right)+c_{3} \mu^{n} n^{3} \\
& \leq c_{1} \mu^{n} n^{\tau+1}+c \mu^{n} n^{3},
\end{aligned}
$$

where $\tau=1$, except for $\alpha=\alpha^{\prime}=1$, in which case $\tau=2$.

5. Convergence analysis. In this section, we establish a convergence result for the approximate solution $\widetilde{v}_{\lambda, n}^{\delta}$ obtained from the numerical method developed in the previous section. We let $\widetilde{v}$ denote the solution of equation (2.6), that is, $\widetilde{v}=\mathcal{K}^{\dagger} h$, where $\mathcal{K}^{\dagger}$ denotes the Moore-Penrose generalized inverse of $\mathcal{K}$. By the triangle inequality, we 
have that

$$
\left\|\widehat{v}-\widetilde{v}_{\lambda, n}^{\delta}\right\|_{\infty} \leq\left\|\widetilde{v}-v_{\lambda}\right\|_{\infty}+\left\|v_{\lambda}-\widetilde{v}_{\lambda, n}^{\delta}\right\|_{\infty},
$$

where $v_{\lambda}$ is the Tikhonov regularization solution of equation (2.6). We shall estimate the two terms on the right hand side of (5.1). To estimate the first term of equation (5.1), we impose the following

(H) $\widehat{v} \in R\left((\mathcal{K} \mathcal{K})^{\nu} \mathcal{K}\right)$ with $0<\nu \leq 1$, that is, there exists an $\omega \in L^{\infty}(E)$ such that $\widehat{v}=(\mathcal{K} \mathcal{K})^{\nu} \mathcal{K} \omega$.

According to [12], we have the following lemma.

Lemma 5.1. If hypothesis $(\mathrm{H})$ holds, then

$$
\left\|\widehat{v}-v_{\lambda}\right\|_{\infty} \leq c(\nu)\|\omega\|_{\infty} \lambda^{\nu}, \quad \text { as } \lambda \rightarrow 0,
$$

where $c(\nu)$ is the constant defined by

$$
c(\nu):= \begin{cases}(\sin \nu \pi) /(\nu(1-\nu) \pi) & \text { if } 0<\nu<1 \\ 1 & \nu=1\end{cases}
$$

We next estimate the second term on the right hand side of (5.1). For this purpose, we write the equivalent operator form of system (4.13). To this end, we let $\widetilde{K}_{n}$ denote the operator from $X_{n}$ to $X_{n}$ such that its matrix representation under the basis $\omega_{i j},(i, j) \in U_{n}$, is $\mathbf{E}_{n}^{-1} \widetilde{\mathbf{K}}_{n}$. Associated with the operator $\widetilde{K}_{n}$, the system (4.13) has the following operator form

$$
\left\{\begin{array}{l}
\widetilde{K}_{n} \widetilde{x}_{\lambda, n}^{\delta}+\sqrt{\lambda} \widetilde{v}_{\lambda, n}^{\delta}=0, \\
\widetilde{K}_{n} \widetilde{v}_{\lambda, n}^{\delta}-\sqrt{\lambda} \widetilde{v}_{\lambda, n}^{\delta}=\mathcal{P}_{n} h^{\delta} .
\end{array}\right.
$$

By eliminating the variable $\widetilde{x}_{\lambda, n}^{\delta}$, we obtain the operator equation of the function $\widetilde{v}_{\lambda, n}^{\delta}$,

$$
(\lambda \mathcal{I}+\widetilde{\mathcal{K}}) \widetilde{v}_{\lambda, n}^{\delta}=\widetilde{K}_{n} \mathcal{P}_{n} h^{\delta} .
$$

To estimate $\left\|v_{\lambda}-\widetilde{v}_{\lambda, n}^{\delta}\right\|^{\infty}$, we study the error $\left\|\mathcal{K}^{2}-\mathcal{K}_{n}^{2}\right\|_{\infty}$, where the operator norm is defined on the space $X_{n}$. By the triangle inequality, we have that

$$
\left\|\mathcal{K}^{2}-\widetilde{\mathcal{K}}_{n}^{2}\right\|_{\infty} \leq\left\|\mathcal{K}^{2}-\mathcal{K}_{n}^{2}\right\|_{\infty}+\left\|\mathcal{K}_{n}^{2}-\widehat{\mathcal{K}}_{n}^{2}\right\|_{\infty}+\left\|\widehat{\mathcal{K}}_{n}^{2}-\widetilde{\mathcal{K}}_{n}^{2}\right\|_{\infty}
$$


We shall estimate the three errors that appear on the right hand side of (5.6).

By the definition of the operator norm on Banach spaces, we have that

$$
\|\mathcal{K}\|_{\infty}=\sup _{x \in X} \frac{\|\mathcal{K} x\|_{\infty}}{\|x\|_{\infty}} \geq \sup _{x \in X_{n}} \frac{\|\mathcal{K} x\|_{\infty}}{\|x\|_{\infty}}=\left\|\left.\mathcal{K}\right|_{X_{n}}\right\|_{\infty} .
$$

Recalling that $\|\mathcal{K}\|_{\infty}$ is bounded, $\left.\mathcal{K}\right|_{X_{n}} \|_{\infty}$ is bounded as well. It follows from properties (v) and (vi) in Proposition 3.1 that a positive constant $c$ exists such that, for all $n \in N$,

$$
\begin{aligned}
\left\|\mathcal{K}^{2}-\mathcal{K}_{n}^{2}\right\|_{\infty} \leq & \left\|\left.\left(\mathcal{I}-\mathcal{P}_{n}\right) \mathcal{K}\right|_{X_{n}}\right\|_{\infty}\left\|\left.\mathcal{K}\right|_{X_{n}}\right\|_{\infty} \\
& +\left\|\left.\mathcal{P}_{n} \mathcal{K}\right|_{X_{n}}\right\|_{\infty}\left\|\left.\left(\mathcal{I}-\mathcal{P}_{n}\right) \mathcal{K}\right|_{X_{n}}\right\|_{\infty} \leq c \mu^{k n}
\end{aligned}
$$

In order to estimate the second item on the right hand of (5.6), we next analyze the convergence of the truncation algorithm.

Our first goal is to obtain estimates for the entries of matrix $\mathbf{K}_{n}$. To this end, we state the following lemma.

Lemma 5.2. There exists a positive constant $c$ such that, for all $n \in N$, all indices $(i, j),\left(i^{\prime}, j^{\prime}\right) \in U_{n}$ and all parameters $\sigma>0$,

$$
\left|K_{i^{\prime} j^{\prime}, i j}\right| \leq c\left(d_{i} d_{i^{\prime}}\right)^{k} \sigma^{-(2 k+1)} \sum_{x \in \hat{S}_{i^{\prime} j^{\prime}}} \int_{S_{i j}}\left|g^{(2 k)}\left(\frac{x-y}{\sigma}\right)\right| \mathrm{d} y .
$$

Proof. The proof of this lemma is essentially the same as that of [20, Lemma 3.1]. Thus, we do not present the details here.

With Lemma 5.2, we have the following lemma.

Lemma 5.3. If $\epsilon_{i^{\prime} i}$ and $\delta_{i^{\prime} i}$ are chosen according to (4.4) and (4.5), respectively, then there exists a positive constant $c$ such that, for all $\sigma>0$

$$
\left\|\mathbf{K}_{i^{\prime} i}-\widehat{\mathbf{K}}_{i^{\prime} i}\right\|_{\infty} \leq c\left(d_{i} d_{i^{\prime}}\right)^{k} \epsilon_{i^{\prime} i} / \sigma^{2 k}
$$


Proof. Define the set $Z_{i^{\prime} i j^{\prime}}:=\left\{j: j \in \omega(i)\right.$ and $\operatorname{dist}\left(S_{i j}, S_{i^{\prime} j^{\prime}}\right)>$ $\left.\sigma \delta_{i^{\prime} i}\right\}$. Note that

$$
\left\|\mathbf{K}_{i^{\prime} i}-\widehat{\mathbf{K}}_{i^{\prime} i}\right\|_{\infty}=\max _{j^{\prime} \in \omega\left(i^{\prime}\right)} \sum_{j \in Z_{i^{\prime} i j^{\prime}}}\left|K_{i^{\prime} j^{\prime}, i j}\right| .
$$

According to Lemma 5.2, we observe that

$$
\begin{aligned}
\left\|\mathbf{K}_{i^{\prime} i}-\widehat{\mathbf{K}}_{i^{\prime} i}\right\|_{\infty} & \leq c\left(d_{i} d_{i}^{\prime}\right)^{k} \sigma^{-(2 k+1)} \\
& \times \max _{j^{\prime} \in \omega\left(i^{\prime}\right)} \sum_{j \in Z_{i^{\prime} i j^{\prime}}} \sum_{x \in \hat{S}_{i^{\prime} j^{\prime}}} \int_{S_{i j}}\left|g^{(2 k)}\left(\frac{(x-y)}{\sigma}\right)\right| \mathrm{d} y .
\end{aligned}
$$

Using a change of variable, $t=(x-y) / \sigma$ in the integral appearing on the right hand side of inequality $(5.8)$, we conclude that

$$
\left\|\mathbf{K}_{i^{\prime} i}-\mathbf{K}_{i^{\prime} i}\right\|_{\infty} \leq c r \sigma^{-2 k}\left(d_{i} d_{i}^{\prime}\right)^{k} \sum_{x \in \hat{S}_{i^{\prime} j^{\prime}}} \int_{\delta_{i^{\prime} i}}^{\infty}\left|g^{(2 k)}(t)\right| \mathrm{d} y
$$

where $r$ is the same constant that appears in Section 3. Since there are finite points in $\widehat{S}_{i^{\prime} j^{\prime}}$, by employing the definition of $\delta_{i^{\prime} i}$, we obtain the desired estimate.

Noting that the matrix representation of operator $\mathcal{K}_{n}$ relative to basis $W_{n}$ is given by $\mathbf{E}_{n}^{-1} \mathbf{K}_{n}$ and $\widehat{\mathbf{K}}_{n}$ is the truncated matrix of $\mathbf{K}_{n}$, we let $\mathcal{K}_{n}$ denote the operator from $X_{n}$ into itself having the matrix representation $\mathbf{E}_{n}^{-1} \mathbf{K}_{n}$ relative to the basis $W_{n}$. Using this notation, we present the next lemma. For $v \in L^{\infty}(E)$, we set

$$
\mathcal{P}_{n} v:=\sum_{(i, j) \in U_{n}} v_{i j} w_{i j}
$$

where the quantities $v_{i j}$ are the linear functionals of $v$, which we defined in Section 3.

Lemma 5.4. If $\alpha$ and $\alpha^{\prime}$ are real numbers, parameters $\epsilon_{i^{\prime} i}$ are chosen as

$$
\epsilon_{i^{\prime} i}:=a^{-\eta} \mu^{-\eta\left(-n+\alpha(n-i)+\alpha^{\prime}\left(n-i^{\prime}\right)\right)} \sigma^{2 k}, \quad i, i^{\prime} \in Z_{n+1}
$$


for some constant $a>0$ and $\eta:=2 k-1$. Then there exists a positive constant $c$ such that, for $v \in W^{k, \infty}(E)$,

$$
\left\|\left(\mathcal{K}_{n}-\widehat{\mathcal{K}}_{n}\right) \mathcal{P}_{n} v\right\|_{\infty} \leq c \mu^{\prime} \mu^{-k n}\|v\|_{k, \infty}
$$

Furthermore,

$$
\left\|\mathcal{K}_{n}-\widehat{\mathcal{K}}_{n}\right\|_{\infty} \leq c \mu^{\prime} \mu^{-k n},
$$

where, for given real numbers $\alpha_{1}, \beta_{1}$ and $n \in N_{0}$,

$$
\mu\left[\alpha_{1}, \beta_{1} ; n\right]:=\sum_{i \in Z_{n+1}} \mu^{\alpha_{1} i} \sum_{i^{\prime} \in Z_{n+1}} \mu^{\beta_{1} i^{\prime}},
$$

and

$$
\mu^{\prime}:=(n+1) \mu\left[2 k-\eta \alpha, k-\eta \alpha^{\prime} ; n\right] \mu^{n} .
$$

Proof. For $\mathbf{v}:=\left[v_{i j}:(i, j) \in U_{n}\right]$, we let $\mathbf{h}:=\mathbf{E}_{n}^{-1}\left(\mathbf{K}_{n} \widehat{\mathbf{K}}_{n}\right) \mathbf{v}$ and write $\mathbf{h}=\left[h_{i j}:(i, j) \in U_{n}\right]$. We then have that

$$
\left(\mathcal{K}_{n}-\widehat{\mathcal{K}}_{n}\right) \mathcal{P}_{n} v=\sum_{(i, j) \in U_{n}} h_{i j} w_{i j} .
$$

By Proposition 3.1 (vii), we conclude that

$$
\left\|\left(\mathcal{K}_{n}-\widehat{\mathcal{K}}_{n}\right) \mathcal{P}_{n} v\right\|_{\infty} \leq \theta_{1}(n+1)\left\|\left(\mathbf{K}_{n}-\widehat{\mathbf{K}}_{n}\right) \mathbf{v}\right\|_{\infty}
$$

We next estimate $\left\|\left(\mathbf{K}_{n}-\widehat{\mathbf{K}}_{n}\right) \mathbf{v}\right\|_{\infty}$. To this end, we introduce the matrix $\boldsymbol{\Delta}_{n}:=\left[\Delta_{i^{\prime} j^{\prime}, i j}:\left(i^{\prime}, j^{\prime}\right),(i, j) \in U_{n}\right]$ whose entries are given by $\Delta_{i^{\prime} j^{\prime}, i j}:=\nu \mu^{k(n-i)+n}\left(K_{i^{\prime} j^{\prime}, i j}-\widehat{K}_{i^{\prime} j^{\prime}, i j}\right)$, where $\nu:=1 / \mu[2 k-\alpha \eta, k-$ $\left.\alpha^{\prime} \eta ; n\right]$. Defining vector $\mathbf{v}^{\prime}:=\left[v_{i j}^{\prime}:(i, j) \in U_{i j}\right]$, with components $v_{i j}^{\prime}:=\mu^{i k} v_{i j},(i, j) \in U_{n}$, we have that

$$
\left\|\left(\mathbf{K}_{n}-\widehat{\mathbf{K}}_{n}\right) \mathbf{v}\right\|_{\infty} \leq \nu^{-1} \mu^{-k n-n}\left\|\boldsymbol{\Delta}_{n}\right\|_{\infty}\left\|\mathbf{v}^{\prime}\right\|_{\infty} .
$$

We next estimate $\left\|\boldsymbol{\Delta}_{n}\right\|_{\infty}$. By the definition of matrix $\Delta_{i^{\prime} j^{\prime}, i j}$, we have that

$$
\sum_{(i, j) \in U_{n}}\left|\Delta_{i^{\prime} j^{\prime}, i j}\right| \leq \nu \sum_{i \in Z_{n+1}} \mu^{k(n-i)+n}\left\|\mathbf{K}_{i^{\prime} i}-\widehat{\mathbf{K}}_{i^{\prime} i}\right\|_{\infty} .
$$

According to Lemma 5.3, there exists a positive constant $c$ such that

$$
\sum_{(i, j) \in U_{n}}\left|\Delta_{i^{\prime} j^{\prime}, i j}\right| \leq c \nu \sum_{i \in Z_{n+1}} \mu^{k(n-i)+n-k\left(i+i^{\prime}\right)} \epsilon_{i^{\prime} i} / \sigma^{2 k} .
$$


Furthermore, using the choice of $\epsilon_{i^{\prime} i}$ in inequality (5.14) yields that

$$
\sum_{(i, j) \in U_{n}}\left|\Delta_{i^{\prime} j^{\prime}, i j}\right| \leq c \nu \alpha^{-\eta} \sum_{i \in Z_{n+1}} \mu^{(2 k-\eta \alpha)(n-i)} \mu^{\left(k-\eta \alpha^{\prime}\right)\left(n-i^{\prime}\right)} .
$$

Since

$$
\begin{aligned}
\sum_{i \in Z_{n+1}} \mu^{(2 k-\eta \alpha)(n-i)} \mu^{\left(k-\eta \alpha^{\prime}\right)\left(n-i^{\prime}\right)} & \leq \sum_{i \in Z_{n+1}} \mu^{(2 k-\eta \alpha) i} \sum_{i \in Z_{n+1}} \mu^{\left(k-\eta \alpha^{\prime}\right) i^{\prime}} \\
& =\mu\left[2 k-\alpha \eta, k-\alpha^{\prime} \eta ; n\right]
\end{aligned}
$$

by the definition of $\nu$, we finally have that

$$
\left\|\boldsymbol{\Delta}_{n}\right\|_{\infty}=\max _{\left(i^{\prime}, j^{\prime}\right) \in U_{n}} \sum_{(i, j) \in U_{n}}\left|\Delta_{i^{\prime} j^{\prime}, i j}\right| \leq c .
$$

Moreover, by [7, Lemma 7.3.1], there also exists a constant $c$ for all $v \in W^{k, \infty}$,

$$
\left\|\mathbf{v}^{\prime}\right\| \leq c\|v\|_{k, \infty}
$$

Combining inequalities (5.12)-(5.16) yields the inequality (5.10).

For the second inequality (5.11), by using the definition of the operator norm in Banach spaces and estimate (5.10), we have that

$$
\begin{aligned}
\left\|\mathcal{K}_{n}-\widehat{\mathcal{K}}_{n}\right\|_{\infty} & =\sup _{v \in X_{n}} \frac{\left\|\left(\mathcal{K}_{n}-\widehat{\mathcal{K}}_{n}\right) \mathcal{P}_{n} v\right\|_{\infty}}{\|v\|_{\infty}} \\
& \leq \sup _{v \in W^{k, \infty}} \frac{\left\|\left(\mathcal{K}_{n}-\widehat{\mathcal{K}}_{n}\right) \mathcal{P}_{n} v\right\|_{\infty}}{\|v\|_{k, \infty}} \leq c \mu^{\prime} \mu^{-k n} .
\end{aligned}
$$

In the next lemma, we translate the second estimate in Lemma 5.4 to an estimate of $\left\|\mathcal{K}_{n}^{2}-\widehat{\mathcal{K}}_{n}^{2}\right\|_{\infty}$.

Lemma 5.5. Suppose that $\alpha$ and $\alpha^{\prime}$ satisfy the conditions

$$
2 k-\eta \alpha=1, k-\eta \alpha^{\prime}<1 \quad \text { and } \quad(2 k-\eta \alpha)+\left(k-\eta \alpha^{\prime}\right)<1 .
$$

If $\epsilon_{i^{\prime} i}$ and $\delta_{i^{\prime} i}$ are chosen according to equations (4.4) and (4.5), respectively, and $\eta=2 k-1$, then there exists a positive constant $c$ such that, for all $n \geq 2$ and $\sigma>0$,

$$
\left\|\mathcal{K}_{n}^{2}-\widehat{\mathcal{K}}_{n}^{2}\right\|_{\infty} \leq c n \mu^{-k n} .
$$


Proof. According to the triangle inequality and inequality (5.11) in Lemma 5.4, we have that

$$
\begin{aligned}
\left\|\mathcal{K}_{n}^{2}-\widehat{\mathcal{K}}_{n}^{2}\right\|_{\infty} & \leq\left\|\mathcal{K}_{n}\right\|_{\infty}\left\|\mathcal{K}_{n}-\widehat{\mathcal{K}}_{n}\right\|_{\infty}+\left\|\mid \mathcal{K}_{n}-\widehat{\mathcal{K}}_{n}\right\|_{\infty}\left\|\widehat{\mathcal{K}}_{n}\right\|_{\infty} \\
& \leq c \mu^{\prime} \mu^{-k n} .
\end{aligned}
$$

Moreover, since $\mu[\alpha, \beta ;](n+1) \mu^{-e n} \sim n$ while $\alpha=e, \beta<e, \alpha+\beta<e$, by the way we choose $\alpha, \alpha^{\prime}$, we have that $\mu^{\prime} \sim n$. Substituting this quantity into the above estimate gives the desired result of this lemma.

Next we turn to estimating the third term $\left\|\widehat{\mathcal{K}}_{n}^{2}-\widetilde{\mathcal{K}}_{n}^{2}\right\|_{\infty}$. This error is caused by the numerical integration used in computing the nonzero entries of the truncated coefficient matrix of the linear system. To this end, for $(i, j),\left(i^{\prime}, j^{\prime}\right) \in U_{n}$, we let

$$
E_{m}\left(h_{i^{\prime} j^{\prime}, i j}\right):=\sum_{x \in \hat{S}_{i^{\prime} j^{\prime}}} c_{x} \sum_{\alpha \in Z_{m^{\prime \prime}}}\left|\int_{Q_{\alpha}} S_{\alpha}\left(h_{i j}\right)(x, y)-h_{i j}(x, y) \mathrm{d} y\right|,
$$

which is the error of the approximation of $K_{i^{\prime} j^{\prime}, i j}$. The following lemma gives an estimate of the error $E_{m}\left(h_{i^{\prime} j^{\prime}, i j}\right)$.

Lemma 5.6. If $\epsilon>0$ is a given fixed number and $m$ is a positive integer, then there exists a positive constant $c$ such that, for all $m, \sigma$, for all $\left(i^{\prime}, j^{\prime}\right),(i, j) \in U_{n}$, for all $\beta$ satisfying (4.12), and for all $0<\sigma<1 / 2$,

$$
E_{m}\left(h_{i^{\prime} j^{\prime}, i j}\right) \leq c \mu^{k(i-1)} \beta^{m} .
$$

Proof. We estimate $E_{m}\left(h_{i^{\prime} j^{\prime}, i j}\right)$ by splitting it into two sums. For $\iota \in Z_{m}$, we introduce two index sets

$$
\Gamma_{\iota}^{r}:=\left\{\alpha \in Z_{m^{\prime \prime}}: Q_{\alpha} \in \Pi\left(h_{i j}\right), Q_{\alpha} \subseteq\left[t_{\iota}^{r}, t_{\iota+1}^{r}\right]\right\}
$$

and

$$
\Gamma_{\iota}^{l}:=\left\{\alpha \in Z_{m^{\prime \prime}}: Q_{\alpha} \in \Pi\left(h_{i j}\right), Q_{\alpha} \subseteq\left[t_{\iota+1}^{l}, t_{\iota}^{l}\right]\right\}
$$


Associated with these two index sets, we set

$$
E_{m, k_{\iota}}^{r}\left(h_{i^{\prime} j^{\prime}, i j}\right):=\sum_{x \in \hat{S}_{i^{\prime} j^{\prime}}} c_{x} \sum_{\alpha \in \Gamma_{\iota}^{r}}\left|\int_{Q_{\alpha}} S_{\alpha}\left(h_{i j}\right)(x, y)-h_{i j}(x, y) \mathrm{d} y\right|
$$

and

$$
E_{m, k_{\iota}}^{l}\left(h_{i^{\prime} j^{\prime}, i j}\right):=\sum_{x \in \hat{S}_{i^{\prime} j^{\prime}}} c_{x} \sum_{\alpha \in \Gamma_{\iota}^{l}}\left|\int_{Q_{\alpha}} S_{\alpha}\left(h_{i j}\right)(x, y)-h_{i j}(x, y) \mathrm{d} y\right| .
$$

Clearly, we have that

$$
E_{m}\left(h_{i^{\prime} j^{\prime}, i j}\right)=\sum_{\iota \in Z_{m}} E_{m, k_{\iota}}^{r}\left(h_{i^{\prime} j^{\prime}, i j}\right)+\sum_{\iota \in Z_{m}} E_{m, k_{\iota}}^{l}\left(h_{i^{\prime} j^{\prime}, i j}\right) .
$$

We next estimate $E_{m, k_{\iota}}^{r}\left(h_{i^{\prime} j^{\prime}, i j}\right)$. Using the standard quadrature formula, we obtain that

$$
\begin{aligned}
E_{m, k_{\iota}}^{r}\left(h_{i^{\prime} j^{\prime}, i j}\right)= & \sum_{x \in \hat{S}_{i^{\prime} j^{\prime}}} c_{x} \sum_{\alpha \in \Gamma_{\iota}^{r}} \frac{1}{\left(2 k_{\iota}\right) !}\left|D_{y}^{2 k_{\iota}} h_{i j}\left(x, \xi_{\alpha}\right)\right| \\
& \left|\int_{Q_{\alpha}}\left(y-\tau_{\alpha}^{0}\right)^{2} \cdots\left(y-\tau_{\alpha}^{k_{\iota-1}}\right)^{2} \mathrm{~d} y\right|
\end{aligned}
$$

where $\xi_{\alpha} \in\left(q_{\alpha}, q_{\alpha+1}\right)$. We begin with estimating the quantity $1 /(2 k \iota) !\left|D_{y}^{2 k_{\iota}} h_{i j}\left(x, \xi_{\alpha}\right)\right|$. Since $h_{i j}(x, y)=K(x, y) \omega_{i j}(y)$, by the Leibnitz formula for the $k_{\iota}$ th derivative of a product of two functions, we observe that

$$
\frac{1}{\left(2 k_{\iota}\right) !}\left|D_{y}^{2 k_{\iota}} h_{i j}\left(x, \xi_{\alpha}\right)\right| \leq \frac{1}{\left(2 k_{\iota}\right) !} \sum_{p=0}^{k_{0}}\left(\begin{array}{c}
2 k_{\iota} \\
p
\end{array}\right)\left|D_{y}^{\left(2 k_{\iota}-p\right)} K\left(x, \xi_{\alpha}\right)\right|\left|\omega_{i j}^{p}\left(\xi_{\alpha}\right)\right|,
$$

where $k_{0}:=\min \left\{2 k_{\iota}, k\right\}$. Furthermore, since

$$
K(x, y)=\sigma^{-1} g\left(\frac{x-y}{\sigma}\right)
$$


by using the property of the Gauss function, we find that

$$
\frac{1}{\left(2 k_{\iota}\right) !}\left(\begin{array}{c}
2 k_{\iota} \\
p
\end{array}\right)\left|D_{y}^{\left(2 k_{\iota}-p\right)} K(x, \xi \alpha)\right| \leq c|\eta|^{-1} \sigma^{-\left(2 k_{\iota}-p\right)}
$$

where

$$
|\eta|=\left|\xi_{\alpha}-x\right| \geq t_{\iota}
$$

For details of the proof of the above inequality, see the proof of $[\mathbf{1 6}$, Theorem 6.1]. To estimate $\omega_{i j}^{(p)}\left(\xi_{\alpha}\right)$, we introduce the constant

$$
\Delta:=\max _{\substack{p \in Z_{k_{0}} \\ l \in Z_{r}}} \sup \left\{\left|\omega_{1 l}^{(p)}(t)\right|: t \in E\right\} .
$$

According to the definition of $\omega_{i j}$, we have for all $t \in E$ that

$$
\left|\omega_{i j}^{(p)}(t)\right|=\left|\mu^{p(i-1)} \omega_{1 j}^{p}\left(\varphi_{\mathbf{s}}^{-1}(t)\right)\right| \leq \Delta \mu^{p(i-1)} .
$$

Combining equations (5.24)-(5.29) and the inequality that

$$
\sum_{p=0}^{k_{0}} \sigma^{-\left(2 k_{\iota}-p\right)} \leq \frac{\sigma^{-2 k_{\iota}}}{1-\sigma}
$$

we obtain that

$$
\begin{aligned}
& E_{m, k_{\iota}}^{r}\left(h_{i^{\prime} j^{\prime}, i j}\right) \\
\leq & \sum_{x \in \hat{S}_{i^{\prime} j^{\prime}}} c_{x} \sum_{\alpha \in \Gamma_{\iota}^{r}} \frac{c \sigma^{-2 k_{\iota}}}{1-\sigma}\left(t_{\iota+1}^{r}-t_{\iota}^{r}\right)^{2 k_{\iota}}\left(q_{\alpha+1}-q_{\alpha}\right)|\eta|^{-1} \Delta \mu^{k(i-1)} .
\end{aligned}
$$

By the construction of the collocation functionals, positive constants $c_{1}$ and $c_{2}$ exist such that

$$
\operatorname{card}\left(\widehat{S}_{i^{\prime} j^{\prime}}\right) \leq c_{1}
$$

and

$$
\max \left\{\max \left\{\left|c_{x}\right|: x \in \widehat{S}_{i^{\prime} j^{\prime}}\right\}:\left(i^{\prime}, j^{\prime}\right) \in U_{n}\right\} \leq c_{2} .
$$

Using (5.32) and the formula

$$
\sum_{\alpha \in \Gamma_{\iota}^{r}}\left(q_{\alpha+1}-q_{\alpha}\right)=t_{\iota+1}^{r}-t_{\iota}^{r}
$$


in (5.31) yields the estimate

(5.33)

$\sum_{\iota \in Z_{m}} E_{m, k_{\iota}}^{r}\left(h_{i^{\prime} j^{\prime}, i j}\right) \leq c c_{1} c_{2} \Delta \mu^{k(i-1)} \sum_{\iota \in Z_{m}} \frac{\sigma^{2 k_{\iota}}}{1-\sigma}\left(t_{\iota+1}^{r}-t_{\iota}^{r}\right)^{2 k_{\iota}}\left(t_{\iota+1}^{r}-t_{\iota}^{r}\right)|\eta|^{-1}$.

For $\iota \neq 0$, from the definition of $t_{\iota}^{r}$ and inequality (5.27), we get that

$$
\left(t_{\iota+1}^{r}-t_{\iota}^{r}\right)^{2 k_{\iota}}|\eta|^{-1} \leq\left(t_{\iota+1}^{r}-t_{\iota}^{r}\right)^{2 k_{\iota}} t_{\iota}^{-1} \leq\left(\frac{1}{\beta}-1\right)^{2 k_{\iota}} t^{2 k_{\iota}-1}
$$

and

$$
\left(t_{\iota+1}^{r}-t_{\iota}^{r}\right)=\left(\frac{1}{\beta}-1\right) \beta^{m-\iota} .
$$

By the choice of $\beta$ in (4.12) and the definition of $k_{\iota}$, we obtain that (5.36)

$$
\left(\frac{1}{\beta}-1\right)^{2 k_{\iota}} \beta^{-\iota} \sigma^{-2 k_{\iota}}=\left(\frac{1-\beta}{\sigma \beta^{1+\iota / 2 k_{\iota}}}\right)^{2 k_{\iota}} \leq\left(\frac{1-\beta}{\sigma \beta^{1+1 / 2 \epsilon}}\right)^{2 k_{\iota}} \leq 1 .
$$

Moreover, when $\iota \geq[1 / 2 \epsilon]+1,2 k_{\iota}-1 \geq 1$, we have that

$$
\begin{aligned}
\sum_{\iota \in Z_{m}}\left(t_{\iota}\right)^{2 k_{\iota}-1} & \leq \sum_{\iota=0}^{[1 / 2 \epsilon]}\left(t_{\iota}\right)^{2 k_{\iota}-1}+\sum_{\iota=[1 / 2 \epsilon]+1}^{m-1}\left(t_{\iota}\right)^{2 k_{\iota}-1} \\
& \leq\left[\frac{1}{2 \epsilon}\right]+\sum_{\iota=0}^{m-1}\left(t_{\iota}\right) \\
& \leq\left[\frac{1}{2 \epsilon}\right]+\frac{\beta}{1-\beta} .
\end{aligned}
$$

Combining (5.23)-(5.37), the fact that $\sigma<1 / 2$ and the choice (4.12) of $\beta$, we get that

$$
E_{m}\left(h_{i^{\prime} j^{\prime}, i j}\right) \leq c\left(\left[\frac{1}{2 \epsilon}\right]+1\right) \mu^{k(i-1)} \beta^{m},
$$

which leads to the desired estimate of this lemma.

The error bound in the next lemma gives an estimate of $\| \widehat{\mathbf{K}}_{i^{\prime} i}-$ $\widetilde{\mathbf{K}}_{i^{\prime} i} \|_{\infty}$. 
Lemma 5.7. If $\epsilon>0$ is a given fixed number and $m$ is a positive integer, then there exists a positive constant $c$ such that, for all $i, i^{\prime} \in$ $Z_{n+1}$, for all $n \in N$ and, for all $0<\sigma<1 / 2$,

$$
\left\|\widehat{\mathbf{K}}_{i^{\prime} i}-\widetilde{\mathbf{K}}_{i^{\prime} i}\right\|_{\infty} \leq c \mu^{i} \mu^{k(i-1)} \beta^{m} .
$$

Proof. Define $\bar{Z}_{i^{\prime} i j^{\prime}}:=\left\{j \in w(i): \operatorname{dist}\left(S_{i j}, S_{i^{\prime} j^{\prime}}\right) \leq \delta_{i^{\prime} i} \sigma\right\}$. By Lemma 5.6, we have, for all $\left(i^{\prime}, j^{\prime}\right) \in Z_{n+1}$ that

$$
\left\|\widehat{K}_{i^{\prime} i}-\widetilde{K}_{i^{\prime} i}\right\|_{\infty}=\max _{j^{\prime} \in \omega\left(i^{\prime}\right)} \sum_{j \in \bar{Z}_{i^{\prime} i j^{\prime}}} E_{m}\left(h_{i^{\prime} j^{\prime}, i j}\right) \leq c \mu^{i} \mu^{k(i-1)} \beta^{m} .
$$

We next choose the integer $m$ used in the numerical quadrature according to the levels $i$ and $i^{\prime}$ so that the resulting approximation solution $\widetilde{v}_{\lambda, n}^{\delta}$ preserves the nearly optimal convergence order. To this end, we use $m_{i^{\prime} i}$ to denote the integer $m$ for different $i, i^{\prime}$ s.

Lemma 5.8. Let $\epsilon>0$ be a given fixed number. If $\beta$ is chosen according to inequality (4.12), $\alpha$ and $\alpha^{\prime}$ are chosen to satisfy inequality (5.18), and $m_{i^{\prime} i}$ are chosen to satisfy

$$
m_{i^{\prime} i} \geq \frac{\log \mu}{\log \beta}\left(-k(i-1)-\left(i+i^{\prime}\right) k-i-\eta\left(n+(n-i) \alpha+\left(n-i^{\prime}\right) \alpha^{\prime}\right)\right),
$$

then there exists a positive constant $c$ such that, for all $n \geq 2$ and for all $0<\sigma<1 / 2$,

$$
\left\|\widehat{\mathcal{K}}_{n}^{2}-\widetilde{\mathcal{K}}_{n}^{2}\right\|_{\infty} \leq c n \mu^{k n} .
$$

Proof. By using the triangle inequality, we have that

$$
\left\|\widehat{\mathcal{K}}_{n}^{2}-\widetilde{\mathcal{K}}_{n}^{2}\right\|^{\infty} \leq\left(\left\|\widehat{\mathcal{K}}_{n}\right\|_{\infty}+\left\|\widetilde{\mathcal{K}}_{n}\right\|_{\infty}\right)\left\|\widehat{\mathcal{K}}_{n}-\widetilde{\mathcal{K}}_{n}\right\|_{\infty} .
$$

It suffices to estimate $\left\|\widehat{\mathcal{K}}_{n}-\widetilde{\mathcal{K}}_{n}\right\|_{\infty}$ and to prove the boundedness of $\left\|\widetilde{\mathcal{K}}_{n}\right\|_{\infty}$.

By Lemma 5.7 with the choice of $\beta$ and $m_{i^{\prime} i}$, we obtain that

$$
\left\|\widehat{\mathbf{K}}_{i^{\prime} i}-\widetilde{\mathbf{K}}_{i^{\prime} i}\right\|_{\infty} \leq c \mu^{-k\left(i+i^{\prime}\right)} \mu^{-\eta\left(-n+(n-i) \alpha+\left(n-i^{\prime}\right) \alpha^{\prime}\right)} .
$$

Using a similar approach as used in the proof of Lemma 5.4, we may translate the above estimate of the matrices to the operators

$$
\left\|\widehat{\mathcal{K}}_{n}-\widetilde{\mathcal{K}}_{n}\right\|_{\infty} \leq c \mu^{\prime} \mu^{-k n} .
$$


Moreover, since

$$
\left\|\widetilde{\mathcal{K}}_{n}\right\|_{\infty} \leq\left\|\widehat{\mathcal{K}}_{n}\right\|_{\infty}+\left\|\widehat{\mathcal{K}}_{n}-\widetilde{\mathcal{K}}_{n}\right\|_{\infty}
$$

and $\left\|\widehat{\mathcal{K}}_{n}\right\|_{\infty}$ is bounded, estimate (5.41) leads to the boundedness of $\left\|\widetilde{\mathcal{K}}_{n}\right\|_{\infty}$. This, combined with inequalities (5.40) and (5.41) yields the desired result of this lemma.

Lemma 5.9. If $\epsilon_{i^{\prime} i}$ and $\delta_{i^{\prime} i}$ are chosen according to (4.4) and (4.5), respectively, $\eta=2 k-1$, and $m_{i^{\prime} i}, \alpha$ and $\alpha^{\prime}$ are chosen as described in Lemma 5.5, then there exists a positive constant $c_{0}$ such that, for all $n \geq 2$ and $0<\sigma<1 / 2$,

$$
\left\|\mathcal{K}^{2}-\widetilde{\mathcal{K}}_{n}^{2}\right\|_{\infty} \leq c_{0} n \mu^{-k n} .
$$

Proof. Estimate (5.42) is obtained directly by combining inequalities (5.7), (5.19) and (5.39).

We now return to the study of the regularization solution. To this end, we define

$$
M:=\sup _{0 \leq x \leq 1}\left(\int_{0}^{1}|K(x, y)|^{2} d y\right)^{1 / 2}
$$

According to $[\mathbf{1 2}, \mathbf{1 3}]$, for each $\lambda>0$, the operator $\left(\lambda I+\mathcal{K}^{2}\right)$ is invertible from $X$ to $X$, and satisfies

$$
\left\|\left(\lambda \mathcal{I}+\mathcal{K}^{2}\right)^{-1}\right\|_{\infty} \leq \frac{\sqrt{\lambda}+M / 2}{\lambda^{3 / 2}}
$$

With this result and Lemma 5.9, we derive the following proposition which is crucial in the error estimate of $\left\|v_{\lambda}-\widetilde{v}_{\lambda, n}^{\delta}\right\|^{\infty}$.

Proposition 5.10. For each $\lambda>0$, if $n \in N$ is chosen to satisfy

$$
n \mu^{-k n} \leq \frac{1}{2 c_{0}} \frac{\lambda^{3 / 2}}{\sqrt{\lambda}+M / 2},
$$

then $\lambda \mathcal{I}+\widetilde{\mathcal{K}}_{n}^{2}: X \rightarrow X$ is invertible and

$$
\left\|\left(\lambda \mathcal{I}+\widetilde{\mathcal{K}}_{n}^{2}\right)^{-1}\right\|_{\infty} \leq 2 \frac{\sqrt{\lambda}+M / 2}{\lambda^{3 / 2}} .
$$


Proof. We use the identity

$$
\lambda \mathcal{I}+\widetilde{\mathcal{K}}_{n}^{2}=\left(\lambda \mathcal{I}+\mathcal{K}^{2}\right)\left[\mathcal{I}+\left(\lambda \mathcal{I}+\mathcal{K}^{2}\right)^{-1}\left(\mathcal{K}_{n}^{2}-\tilde{\mathcal{K}}^{2}\right)\right]
$$

Using the estimate in Lemma 5.9 for $\left\|\mathcal{K}_{n}^{2}-\widetilde{\mathcal{K}}^{2}\right\|_{\infty},\left\|\left(\lambda \mathcal{I}+\mathcal{K}^{2}\right)^{-1}\right\|_{\infty}$ and the assumption on the choice of $n$, we obtain that

$$
\left\|\left(\lambda \mathcal{I}+\mathcal{K}^{2}\right)^{-1}\right\|_{\infty}\left\|\left(\mathcal{K}_{n}^{2}-\widetilde{\mathcal{K}}^{2}\right)\right\|_{\infty} \leq \frac{1}{2}
$$

This implies that $\mathcal{I}+\left(\lambda \mathcal{I}+\mathcal{K}^{2}\right)^{-1}\left(\mathcal{K}^{2}-\widetilde{\mathcal{K}}_{n}^{2}\right)$ is invertible. Hence, $\lambda \mathcal{I}+\widetilde{\mathcal{K}}_{n}^{2}$ is invertible as an operator from $X_{n}$ to $X_{n}$, and

$$
\left(\lambda I+\widetilde{\mathcal{K}}_{n}^{2}\right)^{-1}=\left[\mathcal{I}+\left(\lambda \mathcal{I}+\mathcal{K}^{2}\right)^{-1}\left(\mathcal{K}_{n}^{2}-\widetilde{\mathcal{K}}^{2}\right)\right]^{-1}\left(\lambda \mathcal{I}+\mathcal{K}^{2}\right)^{-1} .
$$

This, together with estimate (5.46), implies that

$$
\left\|\left(\lambda \mathcal{I}+\widetilde{\mathcal{K}}_{n}^{2}\right)^{-1}\right\|_{\infty} \leq \frac{\left\|\left(\lambda \mathcal{I}+\mathcal{K}^{2}\right)^{-1}\right\|_{\infty}}{1-\left\|\left(\lambda \mathcal{I}+\mathcal{K}^{2}\right)^{-1}\right\|_{\infty}}\left\|\mathcal{K}_{n}^{2}-\widetilde{\mathcal{K}}^{2}\right\|_{\infty} \leq \frac{2(\sqrt{\lambda}+M / 2)}{\lambda^{3 / 2}}
$$

proving the desired result.

From (5.44), we see that the choice of $n$ depends on $\lambda$. For this reason, we shall use the notation $\widetilde{v}_{\lambda, n(\lambda)}^{\delta}$ for $\widetilde{v} \delta_{\lambda, n}$ to indicate its dependence on $\lambda$. We next give an estimate of the error $\left\|v_{\lambda}-\widetilde{v}_{\lambda, n(\lambda)}^{\delta}\right\|_{\infty}$.

Lemma 5.11. If $n$ is chosen to satisfy (5.44), then there exists a positive constant $c$ such that, for all $\lambda>0$ and all $n \geq 2$,

$$
\left\|v_{\lambda}-\widetilde{v}_{\lambda, n(\lambda)}^{\delta}\right\|_{\infty} \leq c\left(\frac{\delta}{\lambda}+n \mu^{-k n} \frac{\sqrt{\lambda}+M / 2}{\lambda^{3 / 2}}+\delta n \mu^{-k n} \frac{\sqrt{\lambda}+M / 2}{\lambda^{3 / 2}}\right) .
$$

Proof. By $\widetilde{v}_{\lambda, n(\lambda)}$, we denote the solution of equation (5.5) with $h^{\delta}$ replaced by $h$. Using the triangle inequality, we have that

$$
\left.\left\|v_{\lambda}-\widetilde{v}_{\lambda, n(\lambda)}^{\delta}\right\|_{\infty} \leq\left\|v_{\lambda}-\widetilde{v}_{\lambda, n(\lambda)}\right\|_{\infty}+\| \widetilde{v}_{\lambda, n(\lambda}\right)-\widetilde{v}_{\lambda, n(\lambda)}^{\delta} \|_{\infty} .
$$

We estimate the two terms on the right hand side of (5.48) separately. For the first term, we have that

$$
v_{\lambda}-\widetilde{v}_{\lambda, n(\lambda)}=\left(\lambda \mathcal{I}+\mathcal{K}^{2}\right)^{-1} \mathcal{K} h-\left(\lambda \mathcal{I}+\widetilde{\mathcal{K}}_{n}^{2}\right)^{-1} \widetilde{\mathcal{K}}_{n} \mathcal{P}_{n} h
$$


which can be rewritten as

$$
\begin{aligned}
v_{\lambda}-\widetilde{v}_{\lambda, n(\lambda)}= & {\left[\left(\lambda \mathcal{I}+\mathcal{K}^{2}\right)^{-1}-\left(\lambda \mathcal{I}+\widetilde{\mathcal{K}}_{n}^{2}\right)^{-1}\right] \mathcal{K} h } \\
& +\left(\lambda \mathcal{I}+\widetilde{\mathcal{K}}_{n}^{2}\right)^{-1}\left[\widetilde{\mathcal{K}}_{n}\left(\mathcal{I}-\mathcal{P}_{n}\right) h+\left(\mathcal{K}-\widetilde{\mathcal{K}}_{n}\right) h\right] .
\end{aligned}
$$

By employing the relation $h=\mathcal{K} v$ and introducing

$$
\begin{aligned}
& v_{1}:=\left(\lambda \mathcal{I}+\widetilde{\mathcal{K}}_{n}^{2}\right)^{-1}\left(\widetilde{\mathcal{K}}_{n}^{2}-\mathcal{K}^{2}\right)\left(\lambda \mathcal{I}+\mathcal{K}^{2}\right)^{-1} \mathcal{K}^{2} v, \\
& v_{2}:=\left(\lambda \mathcal{I}+\widetilde{\mathcal{K}}_{n}^{2}\right)^{-1} \widetilde{\mathcal{K}}_{n}\left(\mathcal{I}-\mathcal{P}_{n}\right) \mathcal{K} v,
\end{aligned}
$$

and

$$
v_{3}:=\left(\lambda I+\widetilde{\mathcal{K}}_{n}^{2}\right)^{-1}\left(\mathcal{K}-\widetilde{K}_{n}\right) \mathcal{K} v,
$$

we have that

$$
v_{\lambda}-\widetilde{v}_{\lambda, n(\lambda)}=v_{1}+v_{2}+v_{3} .
$$

By hypothesis $(\mathrm{H}), \widehat{v} \in R(\mathcal{K})$, and thus, we write $\widehat{v}=\mathcal{K} u$ for some $u \in X$. Since, for any positive number $\lambda$, the operator $\lambda \mathcal{I}+\mathcal{K}^{2}$ is invertible and $\left\|\left(\lambda \mathcal{I}+\mathcal{K}^{2}\right)^{-1} \mathcal{K}^{2}\right\|_{2} \leq 1$, it follows that

$$
\begin{aligned}
\left\|\left(\lambda \mathcal{I}+\mathcal{K}^{2}\right)^{-1} \mathcal{K}^{2} \widehat{v}\right\|_{\infty} & \leq\left\|\mathcal{K}\left(\lambda \mathcal{I}+\mathcal{K}^{2}\right)^{-1} \mathcal{K}^{2} u\right\|_{\infty} \\
& \leq\|\mathcal{K}\|_{L^{2}(E) \rightarrow X}\|u\|_{2} .
\end{aligned}
$$

(A similar development may be found in the proof of [9, Lemma 3.4].) Hence, there exists a positive constant $c$ such that, for all $\lambda>0$,

$$
\left\|\left(\lambda \mathcal{I}+\mathcal{K}^{2}\right)^{-1} \mathcal{K}^{2} \widehat{v}\right\|_{\infty} \leq c .
$$

This estimate, together with equations (5.42) and (5.45), ensures that

$$
\left\|v_{1}\right\|_{\infty} \leq c n \mu^{-k n} \frac{\sqrt{\lambda}+M / 2}{\lambda^{3 / 2}} .
$$

Likewise, combining equation (5.45) with Proposition 3.1 (v) and (vi), we conclude that:

$$
\begin{aligned}
\left\|v_{2}\right\|_{\infty} & \leq\left\|\left(\lambda \mathcal{I}+\widetilde{\mathcal{K}}_{n}^{2}\right)^{-1}\right\|_{\infty}\left\|\widetilde{\mathcal{K}}_{n}\right\|_{\infty}\left\|\left(\mathcal{I}-\mathcal{P}_{n}\right) \mathcal{K}\right\|_{\infty}\|v\|_{\infty} \\
& \leq c \mu^{-k n} \frac{\sqrt{\lambda}+M / 2}{\lambda^{3 / 2}}
\end{aligned}
$$


To estimate $v_{3}$, it suffices to estimate $\left\|\mathcal{K}-\widetilde{\mathcal{K}}_{n}\right\|_{\infty}$. By the triangle inequality, we have that

$$
\left\|\mathcal{K}-\widetilde{\mathcal{K}}_{n}\right\|_{\infty} \leq\left\|\mathcal{K}-\mathcal{K}_{n}\right\|_{\infty}+\left\|\mathcal{K}_{n}-\widehat{\mathcal{K}}_{n}\right\|_{\infty}+\left\|\widehat{\mathcal{K}}_{n}-\widetilde{\mathcal{K}}_{n}\right\|_{\infty} .
$$

According to property [9, Proposition 2.3 (2)], we obtain that

$$
\left\|\mathcal{K}_{n}-\widehat{\mathcal{K}}_{n}\right\|_{\infty} \leq c n \mu^{-k n} .
$$

Moreover, by inequality (5.11) in Lemma 5.4, we have that

$$
\left\|\mathcal{K}_{n}-\widetilde{\mathcal{K}}_{n}\right\|_{\infty} \leq c n \mu^{-k n} .
$$

Substituting estimates (5.41), (5.53) and (5.54) into the right hand side of (5.52) yields

$$
\left\|\mathcal{K}-\widetilde{\mathcal{K}}_{n}\right\|_{\infty} \leq c n \mu^{k n} .
$$

This, with the definition of $v_{3}$ leads to the estimate

$$
\begin{aligned}
\left\|v_{3}\right\|_{\infty} & \leq\left\|\left(\lambda \mathcal{I}+\widetilde{\mathcal{K}}_{n}^{2}\right)^{-1}\right\|_{\infty}\left\|\mathcal{K}-\widetilde{\mathcal{K}}_{n}\right\|_{\infty}\|\mathcal{K} v\|_{\infty} \\
& \leq c n \mu^{-k n} \frac{\sqrt{\lambda}+M / 2}{\lambda^{3 / 2}} .
\end{aligned}
$$

Combining (5.49) with estimates (5.50), (5.51) and (5.56) yields

$$
\left\|v_{\lambda}-\widetilde{v}_{\lambda, n(\lambda)}\right\|_{\infty} \leq c n \mu^{-k n} \frac{\sqrt{\lambda}+M / 2}{\lambda^{3 / 2}} .
$$

It remains to estimate the second term on the right-hand side of inequality (5.48). Recalling

$$
\left(\lambda \mathcal{I}+\widetilde{\mathcal{K}}_{n}^{2}\right)\left(\widetilde{v}_{\lambda, n}-\widetilde{v}_{\lambda, n}^{\delta}\right)=\widetilde{\mathcal{K}}_{n} \mathcal{P}_{n}\left(h-h^{\delta}\right),
$$

we rewrite it in the form

$$
\begin{aligned}
& \widetilde{v}_{\lambda, n(\lambda)}-\widetilde{v}_{\lambda, n(\lambda)}^{\delta}=\left(\lambda \mathcal{I}+\widetilde{\mathcal{K}}^{2}\right)^{-1} \\
& \cdot\left[\mathcal{K} \mathcal{P}_{n}\left(h-h^{\delta}\right)+\left(\widetilde{\mathcal{K}}_{n}-\mathcal{K}\right) \mathcal{P}_{n}\left(h-h^{\delta}\right)+\left(\mathcal{K}^{2}-\widetilde{\mathcal{K}}_{n}^{2}\right)\left(\widetilde{v}_{\lambda, n(\lambda)}-\widetilde{v}_{\lambda, n(\lambda)}^{\delta}\right)\right] .
\end{aligned}
$$

It follows from the second estimate of [9, Lemma 2.1] and hypothesis (2.10) that

$$
\left\|\left(\lambda \mathcal{I}+\mathcal{K}^{2}\right)^{-1} \mathcal{K} \mathcal{P}_{n}\left(h-h^{\delta}\right)\right\|_{\infty} \leq M \frac{\delta}{\lambda} .
$$


Proposition 5.10 and estimates (5.42) and (5.55) ensure that there exists a positive constant $c$ such that, for all $\mathrm{n}$,

$$
\left\|\left(\lambda \mathcal{I}+\mathcal{K}^{2}\right)^{-1}\left(\widetilde{\mathcal{K}}_{n}-\mathcal{K}\right) \mathcal{P}_{n}\left(h-h^{\delta}\right)\right\|_{\infty} \leq c n \mu^{-k n}\left(\sqrt{\lambda}+\frac{M}{2}\right) \frac{\delta}{\lambda^{3 / 2}}
$$

and

$\left\|\left(\lambda \mathcal{I}+\mathcal{K}^{2}\right)^{-1}\left(\mathcal{K}^{2}-\widetilde{\mathcal{K}}_{n}^{2}\right)\left(\widetilde{v}_{\lambda, n(\lambda)}-\widetilde{v}_{\lambda, n(\lambda)}^{\delta}\right)\right\|_{\infty} \leq \frac{1}{2}\left\|\widetilde{v}_{\lambda, n(\lambda)}-\widetilde{v}_{\lambda, n(\lambda)}^{\delta}\right\|_{\infty}$.

Combining the above three estimates (5.58), (5.59) and (5.60), we conclude that

$$
\left\|\widetilde{v}_{\lambda, n(\lambda)}-\widetilde{v}_{\lambda, n(\lambda)}^{\delta}\right\|_{\infty} \leq c\left(\frac{\delta}{\lambda}+\delta n \mu^{k n} \frac{\sqrt{\lambda}+M / 2}{\lambda^{3 / 2}}\right) .
$$

Finally, inequalities (5.57) and (5.61) together with (5.48) lead to the desired result of this lemma.

We are now ready to present the main result of this section, which gives an estimate to the error $\left\|\widehat{v}-\widetilde{v}_{\lambda, n(\lambda)}^{\delta}\right\|_{\infty}$.

Theorem 5.12. If $n$ is chosen to satisfy (5.44) and hypothesis $(\mathrm{H})$ holds, then there exists a positive constant $c_{2}$ such that, for all $\lambda>0$ and all $n \geq 2$,

$\left\|\widehat{v}-\widetilde{v}_{\lambda, n(\lambda)}^{\delta}\right\|_{\infty} \leq c_{1} \lambda^{\nu}+c_{2}\left(\frac{\delta}{\lambda}+n \mu^{-k n} \frac{\sqrt{\lambda}+M / 2}{\lambda^{3 / 2}}+\delta n \mu^{-k n} \frac{\sqrt{\lambda}+M / 2}{\lambda^{3 / 2}}\right)$, with $c_{1}:=c(\nu)\|\omega\|_{\infty}$.

Proof. Estimate (5.62) is obtained by substituting (5.2) and (5.47) into the right hand side of inequality (5.1).

Clearly, the error bound on the right hand side of (5.62) has two parts: One (the last two terms on the right hand side of (5.62)) comes from the projection of the solution to the approximate subspace and the other (the first two terms) comes from the ill-posedness and the presence of noise.

We remark that the accuracy of the discrete model is given by the estimate (5.62) with $k=1$. The last two terms on the right hand side of 
(5.62) may be improved if we choose higher order piecewise polynomial approximation.

6. Regularization parameter choice strategies. Solving system (4.13) requires appropriately choosing the regularization parameter $\lambda$. This section is devoted to the description of an a posteriori parameter choice strategy. We shall adopt a general principle developed in [21] with special consideration to our current context.

We now develop the a posteriori parameter choice strategy for the multiscale collocation method. The main idea is to establish the estimate

$$
\left\|\widehat{v}-\widetilde{v}_{\lambda, n(\lambda)}^{\delta}\right\|_{\infty} \leq \varphi(\lambda)+\frac{\delta}{\psi(\lambda)},
$$

from estimate (5.62) presented in Theorem 5.12 for two continuous increasing functions $\varphi$ and $\psi$, so that the general principle of [21] can be applied. For this purpose, we develop a strategy of choosing the level $n$ of the multiscale approximation according to a given $\lambda$. Specifically, for a given $\lambda>0$, we choose $n:=n(\lambda)$ to satisfy condition (5.44) and to make the second term on the right hand side of estimate (5.62) bounded by a constant multiple of $\delta / \lambda$. In other words, we choose $n(\lambda)$ to be the smallest positive integer satisfying the condition

$$
n \mu^{-k n} \leq \min \left\{\frac{\sqrt{\lambda}}{\sqrt{\lambda}+M / 2}, \frac{\delta \sqrt{\lambda}}{\sqrt{\lambda}+M / 2}, \frac{1}{2 c_{0}} \frac{\lambda^{3 / 2}}{\sqrt{\lambda}+M / 2}\right\},
$$

where $c_{0}$ is the constant appearing in (5.44). This leads to the next theorem.

Theorem 6.1. If hypothesis $(\mathrm{H})$ holds and, for a given $\lambda>0, n(\lambda)$ is chosen to be the smallest positive integer that satisfies (6.2), then estimate (6.1) holds with $\varphi(\lambda):=c_{1} \lambda^{\nu}$ and $\psi(\lambda):=\lambda / 3 c_{2}$.

Proof. The desired result is obtained by using the choice of $n$ that is the smallest integer satisfying (6.2) and substituting it into the right hand side of estimate (5.62).

With Theorem 6.1, we are able to apply the general principle presented in [21] to choose the regularization parameter. We choose the optimal parameter $\lambda_{\text {opt }}$ so that the right hand side with $\varphi(\lambda):=c_{1} \lambda^{\nu}$ 
and $\psi(\lambda):=\lambda / 3 c_{2}$ of $(6.1)$ is minimized. This is accomplished when $\varphi(\lambda)=\delta / \psi(\lambda)$, which gives

$$
\lambda=\lambda_{\mathrm{opt}}=(\varphi \psi)^{-1}(\delta) .
$$

Estimate (6.1) with this choice of the parameter becomes

$$
\left\|\widehat{v}-\widetilde{v}_{\lambda, n(\lambda)}^{\delta}\right\|_{\infty} \leq 2 \varphi\left((\varphi \psi)^{-1}(\delta)\right) .
$$

Alternatively, since, for $\lambda>0$, both $\varphi$ and $\psi$ are continuous increasing functions, we observe from (6.1) that

$$
\lambda_{\mathrm{opt}}=\max \left\{\lambda: \varphi(\lambda) \leq \frac{\delta}{\psi(\lambda)}\right\},
$$

or

$$
\lambda_{\mathrm{opt}}=\min \left\{\lambda: \varphi(\lambda) \geq \frac{\delta}{\psi(\lambda)}\right\} .
$$

We now describe a method for finding an approximation of $\lambda_{\text {opt }}$, specifically, we choose a positive integer $N$ and let

$$
\Delta_{N}:=\left\{\lambda_{i}: 0<\lambda_{0}<\lambda_{1}<\cdots<\lambda_{N}\right\},
$$

be a set of $N+1$ distinct positive numbers. The integer $N$ and the $N+1$ distinct positive numbers will be specified later. We then define a finite set

$$
M\left(\Delta_{N}\right):=\left\{\lambda_{i}: \lambda_{i} \in \Delta_{N}, \varphi\left(\lambda_{i}\right) \leq \frac{\delta}{\psi\left(\lambda_{i}\right)}\right\} .
$$

According to (6.4), we find an approximation of the regularization parameter $\lambda_{\text {opt }}$ by

$$
\lambda_{*}=\max \left\{\lambda_{i}: \lambda_{i} \in M\left(\Delta_{N}\right)\right\} .
$$

The method of choosing the regularization parameter $\lambda_{*}$ described above requires knowing both functions $\varphi$ and $\psi$. The function $\varphi$ involves the unknown smoothness order $\nu$ of the integral operator, while $\psi$ only involves a constant $c_{2}$ which can be estimated. Hence, direct use of the set $M\left(\Delta_{N}\right)$ defined in (6.5) is not feasible. We now modify the definition of the set $M\left(\Delta_{N}\right)$ so that the method described above is implementable. Note that, by the triangle inequality and Theorem 6.1 , 
we observe for $\lambda_{i}, \lambda_{j} \in \Delta_{N}$ that

$$
\begin{aligned}
\left\|\widetilde{v}_{\lambda_{i}, n\left(\lambda_{i}\right)}^{\delta}-\widetilde{v}_{\lambda_{j}, n\left(\lambda_{j}\right)}^{\delta}\right\|_{\infty} & \leq\left\|\widehat{v}-\widetilde{v}_{\lambda_{i}, n\left(\lambda_{i}\right)}^{\delta}\right\|_{\infty}+\left\|\widehat{v}-\widetilde{v}_{\lambda_{j}, n\left(\lambda_{j}\right)}^{\delta}\right\|_{\infty} \\
& \leq \varphi\left(\lambda_{i}\right)+\frac{\delta}{\psi\left(\lambda_{i}\right)}+\varphi\left(\lambda_{j}\right)+\frac{\delta}{\psi\left(\lambda_{j}\right)} .
\end{aligned}
$$

In particular, if $\lambda_{i}, \lambda_{j} \in M\left(\Delta_{N}\right)$, then

$$
\left\|\widetilde{v}_{\lambda_{i}, n\left(\lambda_{i}\right)}^{\delta}-\widetilde{v}_{\lambda_{j}, n\left(\lambda_{j}\right)}^{\delta}\right\|_{\infty} \leq \frac{2 \delta}{\psi\left(\lambda_{i}\right)}+\frac{2 \delta}{\psi\left(\lambda_{j}\right)} .
$$

Due to the monotonicity of $\psi$, for $\lambda_{i}, \lambda_{j} \in M\left(\Delta_{N}\right)$ with $\lambda_{i} \geq \lambda_{j}$, we have that

$$
\left\|\widetilde{v}_{\lambda_{i}, n\left(\lambda_{i}\right)}^{\delta}-\widetilde{v}_{\lambda_{j}, n\left(\lambda_{j}\right)}^{\delta}\right\|_{\infty} \leq \frac{4 \delta}{\psi\left(\lambda_{j}\right)} .
$$

The above discussion leads us to introduce the computable set

$$
\text { (6.6) } \begin{aligned}
& M^{+}\left(\Delta_{N}\right) \\
:= & \left\{\lambda_{i} \in \Delta_{N}:\left\|\widetilde{v}_{\lambda_{i}, n\left(\lambda_{i}\right)}^{\delta}-\widetilde{v}_{\lambda_{j}, n\left(\lambda_{j}\right)}^{\delta}\right\|_{\infty} \leq \frac{4 \delta}{\psi\left(\lambda_{j}\right)}, j=0,1, \ldots, i\right\}
\end{aligned}
$$

to replace the set $M\left(\Delta_{N}\right)$ in the method of choosing the parameter $\lambda_{*}$. In fact, it can be seen from the derivation presented above that, for a given positive integer $N$,

$$
M\left(\Delta_{N}\right) \subseteq M^{+}\left(\Delta_{N}\right)
$$

As a result, we observe that

$$
\lambda_{*} \leq \lambda_{+}:=\max \left\{\lambda_{i}: \lambda_{i} \in M^{+}\left(\Delta_{N}\right)\right\},
$$

and the number $\lambda_{+}$may be considered as an approximation of $\lambda_{*}$. This conjecture is proved in the following lemma.

Lemma 6.2. Suppose that hypothesis $(\mathrm{H})$ holds, $M\left(\Delta_{N}\right) \neq \emptyset, \Delta_{N} \backslash$ $M\left(\Delta_{N}\right) \neq \emptyset$, and there exists a positive constant $q$ for all $\lambda_{i} \in \Delta_{N}$, $i=1,2, \ldots, N$,

$$
\psi\left(\lambda_{i}\right) \leq q \psi\left(\lambda_{i 1}\right) .
$$

If, for $\lambda_{+}, n\left(\lambda_{+}\right)$is chosen to be the smallest positive integer that satisfies (6.2) with $\lambda$ replaced by $\lambda_{+}$, then

$$
\left\|\widehat{v}-\widetilde{v}_{\lambda_{+, n\left(\lambda_{+}\right)}^{\delta}}\right\|_{\infty} \leq 6 q \varphi\left((\varphi \psi)^{-1}(\delta)\right) .
$$


Proof. The desired result may be obtained by modifying the proof of [21, Theorem 2.1] and using estimate (6.1). We skip the details of the proof.

According to Theorem 6.1 and Lemma 6.2, we propose the following rule for choosing the a posteriori regularization parameter.

Rule 6.1. A selection strategy of the a posteriori regularization parameter.

- Choose constants $\rho>0, q_{0}>1$ and a positive integer $N$ according to

$$
\rho \delta q_{0}^{N-1} \leq 1<\rho \delta q_{0}^{N}
$$

- Specify the finite set $\Delta_{N}$ by

$$
\Delta_{N}:=\left\{\lambda_{i}:=\rho \delta q_{0}^{i}: i \in Z_{N+1}\right\} .
$$

- For each $\lambda_{i} \in \Delta_{N}$, choose $n\left(\lambda_{i}\right)$ to be the smallest positive integer that satisfies

$$
n\left(\lambda_{i}\right) \mu^{-k n\left(\lambda_{i}\right)} \leq \min \left\{\frac{\sqrt{\lambda_{i}}}{\sqrt{\lambda_{i}}+M / 2}, \frac{\delta \sqrt{\lambda_{i}}}{\sqrt{\lambda_{i}}+M / 2}, \frac{1}{2 c_{0}} \frac{\lambda^{3 / 2}}{\sqrt{\lambda_{i}}+M / 2}\right\} .
$$

- Find $v_{\lambda_{i}, n\left(\lambda_{i}\right)}^{\delta}$ by solving system (4.13).

- Choose

$$
\lambda_{+}=\max \left\{\lambda_{j}: \lambda_{j} \in \Delta_{N},\left\|\widetilde{v}_{\lambda_{j}, n\left(\lambda_{j}\right)}^{\delta}-\widetilde{v}_{\lambda_{i}, n\left(\lambda_{i}\right)}^{\delta}\right\|_{\infty} \leq \frac{12 \delta c_{2}}{\lambda_{i}}, i=0,1, \ldots, j\right\} .
$$

In the next theorem, we present the convergence order of the approximate solution corresponding to the above choice of $\lambda_{+}$.

Theorem 6.3. If hypothesis $(\mathrm{H})$ holds and $\lambda_{+}$is chosen according to Rule 6.1, then the convergence order of the approximate solution $\widetilde{v}_{\lambda_{+}, n\left(\lambda_{+}\right)}^{\delta}$ is given by

$$
\left\|\widehat{v}-\widetilde{v}_{\lambda_{+}, n\left(\lambda_{+}\right)}^{\delta}\right\|_{\infty}=\mathcal{O}\left(\delta^{\nu / \nu+1}\right), \quad \text { as } \delta \rightarrow 0 .
$$

Proof. This theorem can be proved directly by using Lemma 6.2. It suffices to verify the hypothesis of Lemma 6.2. Using the definition of $\Delta_{N}$, we conclude that $\psi\left(\lambda_{i}\right)=q-0 \psi\left(\lambda_{i-1}\right)$. We observe that $\lambda_{N}>1$. 
Thus, for sufficiently small $\delta$, it is easy to see that

$$
c_{1} \rho^{\nu+1} \delta^{\nu} \leq c_{2} \quad \text { and } \quad c_{1} \lambda_{N} \lambda_{N}^{\nu}>c_{2} \delta .
$$

Hence, we observe that

$$
\varphi\left(\lambda_{0}\right) \leq \frac{\delta}{\psi\left(\lambda_{0}\right)} \quad \text { and } \quad \varphi\left(\lambda_{N}\right)>\frac{\delta}{\psi\left(\lambda_{N}\right)} .
$$

From this, we conclude that $\lambda_{0} \in M\left(\Delta_{N}\right)$ and $\lambda_{N} \notin M\left(\Delta_{N}\right)$. Thus, $M\left(\Delta_{N}\right) \neq \emptyset$ and $\Delta_{N} \backslash M\left(\Delta_{N}\right) \neq \emptyset$. As a result, the hypothesis of Lemma 6.2 is satisfied. Therefore,

$$
\left\|\widehat{v}-\widetilde{v}_{\lambda_{+}, n\left(\lambda_{+}\right)}^{\delta}\right\|_{\infty} \leq 6 q_{0} \varphi\left((\varphi \psi(\delta))^{-1}\right)=c_{3} \delta^{\nu / \nu+1},
$$

with $c_{3}:=6 q_{0} c_{1}\left(3 c_{2} / c_{1}\right)^{\nu / \nu+1}$.

7. Numerical experiments. We present in this section numerical examples to demonstrate the performance of the proposed multiscale collocation method for solving the integral equation model for restoration of out-of-focus images. Comparisons of the continuous model with the discrete model in numerical performance were given in [16] for the Galerkin method. It was shown there that the continuous model significantly outperforms the discrete model, especially in the case of image enlargement. Readers interested in the comparisons are referred to [16] for more information in this regard.

The numerical experiments to be presented in this section will focus on testing the proposed collocation method and the choice of regularization parameters. A blurred image is given in a matrix form. Hence, we need to convert it to a bivariate function on a continuous domain. According to [16], we assume that the image to be restored is an intensity function $v$ defined on the continuous domain $E \times E$ and its pixel values are samples of this function, that is,

$$
v(x, y):=\sum_{i, j=0}^{256} \overline{v_{i j}} \psi_{i}(x) \psi_{j}(y), \quad x, y \in E,
$$


where

$(7.2)$

$$
\psi_{\ell}(t):=\left\{\begin{array}{l}
(256 t-(\ell-1)),(\ell-1) / 256<t \leq \ell / 256, \\
((\ell+1)-256 t), \ell / 256<t \leq(\ell+1) / 256, \\
0 \quad \text { otherwise, } t \in E,
\end{array}\right.
$$

for $\ell=0,1, \ldots, 256$. Here we assume that the size of the image is $257 \times 257$. Then the observed image $h$ can by generated by $(2.2)$ with kernel $k$ given by (2.3). Specifically, by using (7.1), we have that

$$
\begin{aligned}
& h(x, y):=\sum_{i, j=0}^{256} \frac{1}{v_{i j}} \int_{0}^{1} \int_{0}^{1} \frac{1}{2 \pi \sigma^{2}} \\
& \times \exp \left(-\frac{\left(x-x^{\prime}\right)^{2}+\left(y-y^{\prime}\right)^{2}}{2 \sigma^{2}}\right) \psi_{i}\left(x^{\prime}\right) \psi_{j}\left(y^{\prime}\right) d y^{\prime} d x^{\prime}, x, y \in E .
\end{aligned}
$$

We shall use the proposed multiscale collocation method to obtain an approximation of $v$ from $h$.

We now describe the approximation spaces $X_{n}$ and the corresponding collocation functional spaces $L_{n}$. Specifically, for each positive integer $n$, we choose $X_{n}$ as the space of piecewise linear polynomials on $E$ with knots at $j / 2 n, j=1,2, \ldots, 2^{n} 1$. Here $\operatorname{dim} X_{n}=2^{n+1}, \mu=2$ and $r=2$, corresponding to the general case described in Section 3 . The space $X_{0}$ has a basis

$$
w_{00}(x):=-3 x+2, \quad w_{01}(x):=3 x-1,
$$

and $W_{1}$ has a basis

$$
\begin{aligned}
& \omega_{10}(x):= \begin{cases}1-9 / 2 x \in[0,1 / 2], \\
3 / 2 x-1 \in(1 / 2,1], \\
0 & \text { otherwise, }\end{cases} \\
& \omega_{11}(x):= \begin{cases}1 / 2-3 / 2 x \in[0,1 / 2], \\
9 / 2 x-7 / 2 \in(1 / 2,1], \\
0 & \text { otherwise. }\end{cases}
\end{aligned}
$$

The basis functions of the space $W_{i}:=\operatorname{span}\left\{w_{i j}: j \in Z^{2^{i}}\right\}$ can be generated recursively from $W_{1}$ according to the construction described in Section 3. The collocation functionals corresponding to $X_{n}$ are 
constructed likewise. Specifically, we let $\ell_{00}:=\delta_{1 / 3}, \ell_{01}:=\delta_{2 / 3}$ and

$$
\ell_{10}:=-\frac{3}{2} \delta_{1 / 3}+\frac{1}{2} \delta_{2 / 3}+\delta_{1 / 6}, \quad \ell_{11}:=\frac{1}{2} \delta_{1 / 3}-\frac{3}{2} \delta_{2 / 3}+\delta_{5 / 6} .
$$

The functionals $\ell_{i j}$ can be generated recursively from $\ell_{1} 0$ and $\ell_{1} 1$ according to the construction described in Section 3. The approximation spaces and the corresponding collocation functionals have the properties presented in Proposition 3.1.

Applying the multiscale collocation method with the above basis functions and collocation functionals to the system of integral equations obtained from the Tikhonov regularization method, using the matrix compression strategy and the numerical integration scheme introduced in Section 4 yield the linear system (4.13). Since, in the context of image restoration, the size of the matrix is large, the resulting linear system is ill-conditioned. To overcome the ill-conditionedness, we introduce an additional parameter $\lambda_{2}$ and use the following system

$$
\left[\begin{array}{cc}
\widetilde{\mathbf{K}}_{n}+\lambda_{2} \mathbf{E}_{n} & -\sqrt{\lambda_{1}} \mathbf{E}_{n} \\
\sqrt{\lambda_{1}} \mathbf{E}_{n} & \widetilde{\mathbf{K}}_{n}+\lambda_{2} \mathbf{E}_{n}
\end{array}\right]\left[\begin{array}{c}
\widetilde{v}_{\lambda, n}^{\delta} \\
\widetilde{x}_{\lambda, n}^{\delta}
\end{array}\right]=\left[\begin{array}{c}
\mathbf{h}_{n}^{\delta} \\
0
\end{array}\right]
$$

to replace system (4.13). The corresponding operator form of equation (7.6) is

$$
\left(\lambda_{1} \mathcal{I}+\left(\lambda_{2} \mathcal{I}+\widetilde{\mathcal{K}}_{n}\right)\left(\lambda_{2} \mathcal{I}+\mathcal{K}_{n}\right)\right) \widetilde{v}_{\lambda, n}=\left(\lambda_{2} \mathcal{I}+\widetilde{\mathcal{K}}_{n}\right) h^{\delta} .
$$

This requires us to carefully balance the two parameters $\lambda_{1}$ and $\lambda_{2}$ in the preceding integral equation to avoid the ill-conditionedness of the resulting matrix.

In our experiments, we shall test the proposed methods for the white and black Resolution Chart images because, for these images, we do not have to consider their boundary conditions. In our future projects, we shall consider boundary conditions of the images and modify the proposed methods accordingly. Specifically, two original images, the white Resolution Chart and black Resolution Chart of size $257 \times 257$ shown, respectively, in Figure 1 (a) and Figure 2 (a) are used in our experiments. The quality of restored images by our proposed method is evaluated by the peak signal-to-noise ratio (PSNR).

The first experiment is for the noise-free case. We solve the corresponding integral equation by using the proposed multilevel colloca- 
tion method with the piecewise linear polynomial basis functions at level $n=7$. In this case, both parameters $\lambda_{1}$ and $\lambda_{2}$ are chosen so that the method gives the best quality reconstructed image. For the white Resolution Chart, its blurred image with the blurring kernel of $\sigma=0.01$ shown in Figure 1 (b) has the PSNR value $14.69 \mathrm{~dB}$, and the reconstructed image with $\left(\lambda_{1}, \lambda_{2}\right)=(5 e 5,0.00015)$ shown in Figure 1 (c) has the PSNR value $18.80 \mathrm{~dB}$. For the black Resolution Chart, its blurred image with the blurring kernel of $\sigma=0.01$ shown in Figure 2 (b) has the PSNR value $14.94 \mathrm{~dB}$, and the reconstructed image with $\left(\lambda_{1}, \lambda_{2}\right)=(1 e-6,0.00015)$ shown in Figure 1 (c) has the PSNR value $18.99 \mathrm{~dB}$. Specifically, see Tables 1 and 2 .

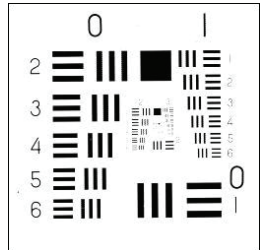

(a)

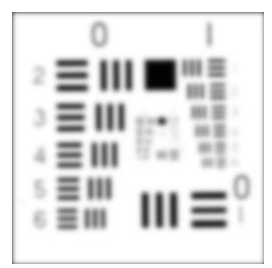

(b)

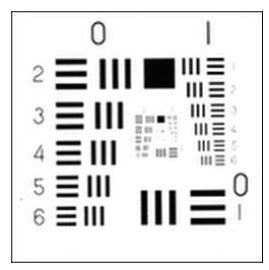

(c)

FIguRE 1. (a) The original white image. (b) Samplings of the continuous observed white image at a uniform grid (with $\sigma=0.01$ in the blurring kernel). (c) The reconstructed white image (with $\lambda_{1}=5 e-5, \lambda_{2}=0.00015$ ).

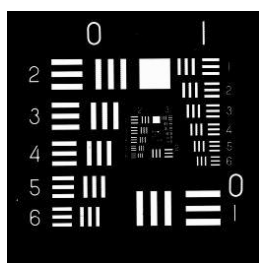

(a)

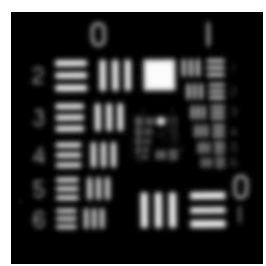

(b)

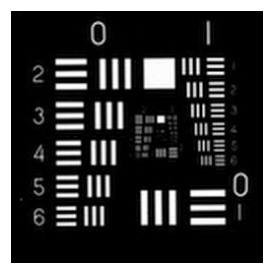

(c)

Figure 2. (a) The original black image. (b) Samplings of the continuous observed black image at a uniform grid (with $\sigma=0.01$ in the blurring kernel). (c) The reconstructed black image (with $\lambda_{1}=1 e-6$, $\left.\lambda_{2}=0.00015\right)$. 
TABLE 1. The PNSR values (dB) for the noise free white 'Resolution Chart' image

\begin{tabular}{lcc}
\hline Noise & blurred image & image reconstructed by proposed method \\
\hline Free & 14.69 & 18.80 \\
\hline
\end{tabular}

TABLE 2. The PNSR values (dB) for the noise free black 'Resolution Chart' image

\begin{tabular}{lcc}
\hline Noise & blurred image & image reconstructed by proposed method \\
\hline Free & 14.94 & 18.99 \\
\hline
\end{tabular}

TABLE 3. The PSNR values $(\mathrm{dB})$ of the images reconstructed with different regularization parameter values from the noisy white 'Resolution Chart' image with noise bound $\delta<0.001$.

\begin{tabular}{cccc}
\hline$\lambda_{1}$ & $\lambda_{2}$ & $n$ & $\begin{array}{c}\text { images reconstructed } \\
\text { by proposed method }\end{array}$ \\
\hline 0.0003 & 0.0003 & 7 & 17.89 \\
0.000648 & 0.0004 & 7 & 18.14 \\
0.0009 & 0.0008 & 7 & 18.12 \\
\hline
\end{tabular}

The second experiment is for the noisy case with noise bound $\delta=0.001$. For the white image, by choosing $\lambda_{1}=\lambda_{+}$according to Rule 6.1 with $\rho:=0.008$ and $q_{0}:=3$, we obtain $n=7, \lambda_{1}=0.000648$. For the black figure, we choose $\rho:=0.02$ and $q_{0}:=3.5$ in Rule 6.1 and obtain the values $n=7$ and $\lambda_{1}=0.000857$. We then compare the PSNR values of the images reconstructed by using our proposed method with these parameter values to those with the other two different parameter values. The numerical results are shown in Tables 3 and 4 and the corresponding reconstructed images are shown in Figures 3 and 4, respectively, for the white and black images. Clearly, from the tables and figures we see that the numerical results and quality of the images obtained with the parameters chosen according to Rule 6.1 are comparable to those obtained in the first example, which are obtained 


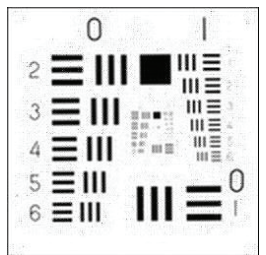

(a)

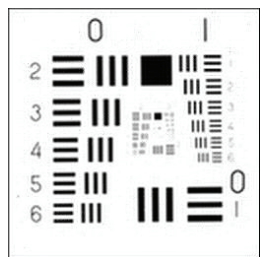

(b)

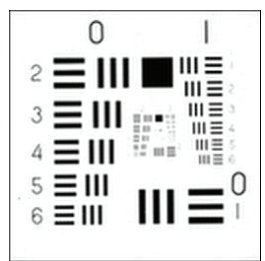

(c)

FIGURE 3. Reconstructed white images for the noisy case (with $\sigma=0.01$ in the blurring kernel and noise bound $\delta=0.001$ ). (a) The reconstructed white image with $\lambda_{1}=0.0003, \lambda_{2}=0.0003$. (b) The reconstructed white image with $\lambda_{1}=0.000648, \lambda_{2}=0.0004$. (c) The reconstructed white image with $\lambda_{1}=0.0009, \lambda_{2}=0.0008$.

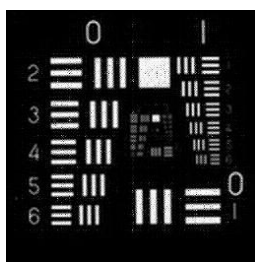

(a)

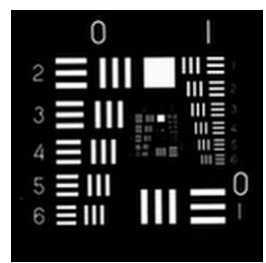

(b)

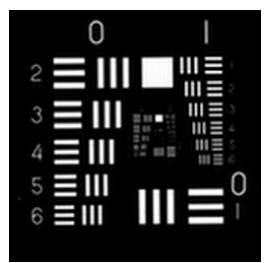

(c)

FIGURE 4. The reconstructed black images for the noisy case (with $\sigma=0.01$ in the blurring kernel and noise bound $\delta=0.001$ ). (a) The reconstructed black image with $\lambda_{1}=0.00015, \lambda_{2}=0.0003$. (b) The reconstructed black image with $\lambda_{1}=0.000857, \lambda_{2}=0.0007$. (c) The reconstructed white image with $\lambda_{1}=0.0012, \lambda_{2}=0.0009$.

TABLE 4. The PSNR values $(\mathrm{dB})$ of the images reconstructed with different regularization parameter values from the noisy black 'Resolution Chart' image with noise bound $\delta<0.001$.

\begin{tabular}{cccc}
\hline$\lambda_{1}$ & $\lambda_{2}$ & $n$ & $\begin{array}{c}\text { images reconstructed } \\
\text { by proposed method }\end{array}$ \\
\hline 0.00015 & 0.0003 & 7 & 18.35 \\
0.000857 & 0.0007 & 7 & 18.82 \\
0.0012 & 0.0009 & 7 & 18.80 \\
\hline
\end{tabular}


by using the best possible parameters, and they are better than those obtained with other choices of the parameters.

\section{REFERENCES}

1. P.M. Anselone, Compact operator approximation theory and applications to integral equations, Prentice-Hill, Englewood Cliffs, 1995.

2. M. Bertero, P. Brianzi and E. Pike, Super-resolution in confocal scanning microcopy, Inv. Prob. 3 (1987), 195-212.

3. Z. Chen, S. Ding, Y. Xu and H. Yang, Multiscale collocation methods for ill-posed integral equations via a coupled system, Inv. Prob. 28 (2012), 025006.

4. Z. Chen, C.A. Micchelli and Y. Xu, A construction of interpolating wavelets on invariant sets, Math. Comp. 68 (1999), 1569-1587.

5. , The Petrov-Galerkin methods for second kind integral equations II: Multiwavelet scheme, Adv. Comp. Math. 7 (1997), 199-233.

6. , Fast collocation method for second kind integral equation, SIAM J. Num. Anal. 40 (2002), 49-55.

7. Z. Chen, C.A. Micchelli and Y. Xu, Multiscale methods for Fredholm integral equations, Cambrige University Press, Cambrige, 2015.

8. Z. Chen, B. Wu and Y. Xu, Error control strategies for numerical integration in fast collocation methods, Northeast Math. J. 21 (2005), 233-252.

9. Z. Chen, Y. Xu and H. Yang, Fast collocation method for solving ill-posed integral equation of the first kind, Inv. Prob. 24 (2008), 065007.

10. W. Fang and M. Lu, A fast collocation method for an inverse boundary value problem, Inter. J. Numer. Meth. Engin. 59 (2004), 1563-1585.

11. R. Gonzalez and R. Woods, Digital image processing, Addison-Wesley, Boston, 1993.

12. W.C. Groetsch, Uniform convergence of regularization methods for Fredholm equations of the first kind, J. Austr. Math. Soc. 39 (1985), 282-286.

13. , The theory of Tikhonov regularization for Fredholm equation of the first kind, Res. Notes Math. 105, Pitman, Boston, 1984.

14. C.P. Hansen, Deconvolution and regularization with Topelitz matrices, Numer. Alg. 29 (2002), 323-378.

15. H. Kaneko and Y. Xu, Gauss-type quadratures for weakly singular integral and their application to Fredholm integral equations of the second kind, Math. Comp. 62 (1994), 739-753.

16. $\mathrm{Y} . \mathrm{Lu}, \mathrm{L}$. Shen and $\mathrm{Y} . \mathrm{Xu}$, Integral equation models for image restoration: High accuracy methods and fast algorithms, Inv. Prob. 26 (2010), 045006.

17. S.V. Maass, P. Pereverzev, R. Ramlau and S.G. Soodky, An adaptive discretization for Tikhonov-Phillips regularizaton with a posteriori parameter selection, Numer. Math. 87 (2001), 485-502. 
18. C.A. Micchelli and $\mathrm{Y} . \mathrm{Xu}$, Using the matrix refinement equation for the construction of wavelets on invariant sets, Appl. Comp. Harmonic Anal. 1 (1994), $344-375$.

19. , Reconstruction and decomposition algorithms for biorthogonal multiwalveles, Multidim. Syst. Signal Proc. 8 (1997), 31-69.

20. C.A. Micchelli, Y. Xu and Y. Zhao, Galerkin methods for second-kind integral equations, J. Comp. Appl. Math. 86 (1997), 251-270.

21. S. Pereverzev and E. Shock, On the adaptive selection of the parameter in regularization of ill-posed problems, SIAM J. Numer. Anal. 43 (2005), 2060-2076.

22. R. Plato, Iterative and parametric methods for linear ill-posed equation Habilitationsschrift, Fachb. Math., Berlin, 1995.

23. _ The Galerkin scheme for Lavrentiev's m-times iterated method to solve the linear accretive Volterra integral equations of the first kind, BIT 37 (1997), 404-423.

24. R. Plato and G. Vainikko, On the regularization of projection methods for solving ill-posed problems, Numer. Math. 57 (1990), 63-79.

25. P.M. Rajan, Convergence anaylysis of a regularized approximation for solving Fredholm integral equations of the first kind, J. Math. Anal. Appl. 279 (2003), $522-530$.

26. U. Tautenhahn, On the method of Lavrentiev regularization for nonlinear ill-posed problem, Inv. Prob. 18 (2002), 191-207.

27. A. Tikhonov and V. Arsenin, Solution to ill-posed problems, Wiley, New York, 1977.

Guangdong Provincial Key Lab of Computational Science and School of Applied Computational Sciences, Sun Yat-sen University, Guangzhou, 510275, P.R. ChINA

\section{Email address: liuyuzh1988@icloud.com}

Department of Mathematics, Syracuse University, Syracuse, NY 13244

Email address: lshen03@syr.edu

Guangdong Provincial Key Lab of Computational Science and School of Applied Computational Sciences, Sun Yat-sen University, Guangzhou, 510275, P.R. China and Department of Mathematics, Syracuse University, SyRaCUSE, NY 13244

Email address: yxu06@syr.edu

Guangdong Provincial Key Lab of Computational Science and School of Applied Computational Sciences, Sun Yat-sen University, Guangzhou, 510275, P.R. CHINA

Email address: mcsyhq@mail.sysu.edu.cn 